\title{
A matched space for time scales and applications to the study on functions
}

\author{
Chao Wang ${ }^{1,2^{*}}$ (D), Ravi P Agarwal ${ }^{2 \dagger}$ and Donal O'Regan ${ }^{3 \dagger}$
}

"Correspondence:

chaowang@ynu.edu.cn

${ }^{1}$ Department of Mathematics,

Yunnan University, Kunming,

Yunnan 650091, People's Republic

of China

2 Department of Mathematics, Texas

A\&M University-Kingsville, 700

University Blvd., Kingsville, TX

78363-8202, USA

Full list of author information is available at the end of the article

${ }^{\dagger}$ Equal contributors

\begin{abstract}
In this paper, using the algebraic structure of the Abelian group, we introduce the concept of a matched space for time scales, and we construct the algebraic structure of matched spaces to solve the closedness of time scales under non-translational shifts. Using a matched space for time scales, a new concept of periodic time scales is introduced. Based on it, new concepts of periodic functions, almost periodic functions and almost automorphic functions whose concepts were defined on translations of their arguments are proposed through non-translational shifts. The results in this paper provide new methods to consider periodic solution, almost periodic solution and almost automorphic solutions for q-difference equations and others on irregular time scales via the background of the algebraic structure.
\end{abstract}

MSC: 26E70; 33E30; 39A13; 34N05; 34C25; 34C27; 43A60

Keywords: time scales; matched space; periodic functions; almost periodic functions; almost automorphic functions; solutions for dynamic equations

\section{Introduction}

Periodic, almost periodic and almost automorphic functions are important classes of functions which arise naturally in differential, difference and integral equations (see [1-6]). The theory of time scales is a useful tool for unifying difference and differential equations (see [7-9] and the references therein). Using the classical definition of periodic time scales (see Kaufmann and Raffoul [10], Akhmet and Turan [11]), periodic solutions, almost periodic solutions and almost automorphic solutions for dynamic equations were considered. In 2006, the authors studied almost periodic solutions of differential equations on certain time scales with transition conditions (DETC) through $\psi$-substitution (see [11]), while the next investigation of almost periodic problems on time scales for impulsive dynamic equations was conducted in 2014 through $\Delta$-calculus directly (see [12-14]). As a matter of fact, the shift closedness of time scales plays a very important role in introducing the welldefined functions on time scales, it is a prerequisite to introduce the functions which are defined by shifts, e.g., periodic functions, almost periodic functions and almost automorphic functions, etc. We note that classical periodic time scales have a very nice translation closedness which paves the way for defining functions through translations. We restate the following definitions and introductory examples which can be found in $[10,11]$.

(c) The Author(s) 2017. This article is distributed under the terms of the Creative Commons Attribution 4.0 International License (http://creativecommons.org/licenses/by/4.0/), which permits unrestricted use, distribution, and reproduction in any medium, provided you give appropriate credit to the original author(s) and the source, provide a link to the Creative Commons license, and indicate if changes were made. 
Definition 1.1 A time scale $\mathbb{T}$ is said to be periodic if there exists $P>0$ such that $t \pm P \in \mathbb{T}$ for all $t \in \mathbb{T}$. If $\mathbb{T} \neq \mathbb{R}$, the smallest positive $P$ is called the period of the time scale.

Example 1.1 The following time scales are periodic.

(1) $\mathbb{T}=h \mathbb{Z}$ has period $P=h$.

(2) $\mathbb{T}=\left\{t=k-q^{m}: k \in \mathbb{Z}, m \in \mathbb{N}_{0}\right\}$, where $0<q<1$, has period $P=1$.

In [15], Adivar proposed a new concept of periodic time scales under shift operators, and this approach enables one to investigate this periodicity notion on a large class of time scales. For example, periodic solutions for $q$-difference equations could be considered since the time scale

$$
\overline{q^{\mathbb{Z}}}=\left\{q^{n}: q>1 \text { is a constant and } n \in \mathbb{Z}\right\} \cup\{0\}
$$

belongs to periodic time scales introduced by Adivar. Introducing the shift operators $\delta_{ \pm}$ on time scales (Definition 4, [15]), the author proposed the following definition.

Definition 1.2 Let $\mathbb{T}$ be a time scale with the shift operators $\delta_{ \pm}$associated with the initial point $t_{0} \in \mathbb{T}^{*}$. The time scale $\mathbb{T}$ is said to be periodic in shifts $\delta_{ \pm}$if there exists $p \in\left(t_{0}, \infty\right)_{\mathbb{T}^{*}}$ such that $(p, t) \in \mathcal{D}_{\mp}$ for all $t \in \mathbb{T}^{*}$. Furthermore, if

$$
P:=\inf \left\{p \in\left(t_{0}, \infty\right)_{\mathbb{T}^{*}}:(p, t) \in \mathcal{D}_{\mp} \text { for all } t \in \mathbb{T}^{*}\right\} \neq t_{0},
$$

then $P$ is called the period of the time scale $\mathbb{T}$, where $\mathcal{D}_{ \pm}=\left\{(s, t) \in\left[t_{0}, \infty\right)_{\mathbb{T}} \times \mathbb{T}^{*}: \delta_{ \pm}(s, t) \in\right.$ $\left.\mathbb{T}^{*}\right\}$.

Note that (1) satisfies Definition 1.2 by taking $t_{0}=1, \mathbb{T}^{*}=q^{\mathbb{Z}}, P=q>1$, and one should note that (2) must satisfy the requirement that

$$
t_{0} \neq P:=\inf \left\{p \in\left(t_{0}, \infty\right)_{\mathbb{T}^{*}}:(p, t) \in \mathcal{D}_{\mp} \text { for all } t \in \mathbb{T}^{*}\right\} \in \mathbb{T},
$$

which indicates that the period $P$ of the time scale is taken from $\mathbb{T}$. Nevertheless, we provide the following example to demonstrate that not all periodic time scales will satisfy Definition 1.2.

Example 1.2 Consider the following time scale, where $a, b>0$ :

$$
\mathbb{P}_{a, b}=\bigcup_{k=-\infty}^{\infty}[(2 k+1)(a+b),(2 k+1)(a+b)+a]
$$

This is a classical periodic time scale with period $P=2(a+b) \notin \mathbb{P}_{a, b}$ and its period set $\Pi=\{2 n(a+b), n \in \mathbb{Z}\}$ satisfying $\mathbb{P}_{a, b} \cap \Pi=\emptyset$. For a particular case, let $a=b=1$, to obtain the time scale

$$
\mathbb{T}=\mathbb{P}_{1,1}=\bigcup_{k=-\infty}^{\infty}[4 k+2,4 k+3]
$$

Clearly $4 n \notin \mathbb{P}_{1,1}$ for $n \in \mathbb{Z}$. Since its period set $\Pi=\{4 n, n \in \mathbb{Z}\}, P=4$, we have $\mathbb{P}_{a, b} \cap \Pi=\emptyset$. 
Wang and Agarwal proposed Example 1.2 in [16] and corrected the concept of almost periodic functions on periodic time scales. Note that Example 1.2 satisfies Definition 1.1 but does not satisfy Definition 1.2 since $P \notin \mathbb{P}_{a, b}$ though $t \pm P \in \mathbb{P}_{a, b}$. As a result, Definition 1.2 does not include Definition 1.1. In [17], the authors introduced some new concepts of periodic time scales attached with a "translation direction". In addition, several examples were provided to show that these new concepts are more general than Definition 1.1, and the composition theorem of time scales from $[17,18]$ will be guaranteed under these new notions. Moreover, the authors of $[19,20]$ proposed and solved the almost periodic problems for $q$-dynamic equations on the quantum time scale for the first time, and the authors introduced the theory of almost periodic functions under shift operators which can derive and include the almost periodic theory of $q$-dynamic equations on the quantum time scale. Also, some new almost periodic functions were constructed on irregular time scales under the stochastic background. Furthermore, in the literature [21], the authors made some significant comments and notes on their related works and the recent development of time scales to contribute to solving the closedness problems on various types of time scales.

The closedness of time scales under shifts is a key and difficult problem which needs to be solved since it is closely related to defining and studying functions, investigating delay dynamic equations on time scales and nonlinear periodic or non-periodic phenomena in the real world. In this paper, we initiate the idea that the shift number set which controls the shifts of a time scale and maintains its shift closedness may be separated from the time scale (e.g., $\mathbb{T} \cap \Pi=\emptyset$ in Example 1.2). Using the algebraic structure of an Abelian group, the concept of a matched space for time scales is introduced and the algebraic structure of matched spaces is constructed to solve the closedness of time scales under nontranslational shifts. With this algebraic structure, a new concept of periodic time scales is introduced and based on it, new concepts of periodic functions, almost periodic functions and almost automorphic functions are proposed through non-translational shifts. Furthermore, these notions attached with shift directions are also introduced and studied.

\section{A matched space for time scales}

In this section, we introduce the algebraic structure of matched spaces for time scales. For details on time scales, we refer the reader to [7-9].

Let $\mathbb{T}$ be a nonempty closed subset (time scale) of $\mathbb{R}$. The forward and backward jump operators $\sigma, \rho: \mathbb{T} \rightarrow \mathbb{T}$ and the graininess $\mu: \mathbb{T} \rightarrow \mathbb{R}^{+}$are defined, respectively, by

$$
\sigma(t)=\inf \{s \in \mathbb{T}: s>t\}, \quad \rho(t)=\sup \{s \in \mathbb{T}: s<t\}, \quad \mu(t)=\sigma(t)-t .
$$

A point $t \in \mathbb{T}$ is called left-dense if $t>\inf \mathbb{T}$ and $\rho(t)=t$, left-scattered if $\rho(t)<t$, rightdense if $t<\sup \mathbb{T}$ and $\sigma(t)=t$, and right-scattered if $\sigma(t)>t$. If $\mathbb{T}$ has a left-scattered maximum $M$, then $\mathbb{T}^{k}=\mathbb{T} \backslash\{M\}$; otherwise $\mathbb{T}^{k}=\mathbb{T}$. If $\mathbb{T}$ has a right-scattered minimum $m$, then $\mathbb{T}_{k}=\mathbb{T} \backslash\{m\}$; otherwise $\mathbb{T}_{k}=\mathbb{T}$.

A function $f: \mathbb{T} \rightarrow \mathbb{R}$ is right-dense continuous provided it is continuous at a right-dense point in $\mathbb{T}$ and its left-side limits exist at left-dense points in $\mathbb{T}$. If $f$ is continuous at each right-dense point and each left-dense point, then $f$ is said to be a continuous function on $\mathbb{T}$. 
For $y: \mathbb{T} \rightarrow \mathbb{R}$ and $t \in \mathbb{T}^{k}$, we define the delta derivative of $y(t), y^{\Delta}(t)$, to be the number (if it exists) with the property that for a given $\varepsilon>0$, there exists a neighborhood $U$ of $t$ such that

$$
\left|[y(\sigma(t))-y(s)]-y^{\Delta}(t)[\sigma(t)-s]\right|<\varepsilon|\sigma(t)-s|
$$

for all $s \in U$.

Now, we construct the algebraic structure for a pair $\left(\Pi^{*}, \tilde{\delta}\right)$ by using an Abelian group property to introduce the definition of a relatively dense set with respect to the group $\left(\Pi^{*}, \tilde{\delta}\right)$, where $\Pi^{*}$ is a subset of $\mathbb{R}$ together with an operation $\tilde{\delta}$.

Definition 2.1 Let $\Pi^{*}$ be a subset of $\mathbb{R}$ together with an operation $\tilde{\delta}$, and let a pair $\left(\Pi^{*}, \tilde{\delta}\right)$ be an Abelian group and $\tilde{\delta}$ be increasing with respect to its second argument, i.e., $\Pi^{*}$ and $\tilde{\delta}$ satisfy the following conditions:

(1) $\Pi^{*}$ is closed with respect to an operation $\tilde{\delta}$, i.e., for any $\tau_{1}, \tau_{2} \in \Pi^{*}$, we have $\tilde{\delta}\left(\tau_{1}, \tau_{2}\right) \in \Pi^{*}$.

(2) For any $\tau \in \Pi^{*}$, there exists an identity element $e_{\Pi^{*}} \in \Pi^{*}$ such that $\tilde{\delta}\left(e_{\Pi^{*}}, \tau\right)=\tau$.

(3) For all $\tau_{1}, \tau_{2}, \tau_{3} \in \Pi^{*}, \tilde{\delta}\left(\tau_{1}, \tilde{\delta}\left(\tau_{2}, \tau_{3}\right)\right)=\tilde{\delta}\left(\tilde{\delta}\left(\tau_{1}, \tau_{2}\right), \tau_{3}\right)$ and $\tilde{\delta}\left(\tau_{1}, \tau_{2}\right)=\tilde{\delta}\left(\tau_{2}, \tau_{1}\right)$.

(4) For each $\tau \in \Pi^{*}$, there exists an element $\tau^{-1} \in \Pi^{*}$ such that $\tilde{\delta}\left(\tau, \tau^{-1}\right)=\tilde{\delta}\left(\tau^{-1}, \tau\right)=e_{\Pi^{*}}$, where $e_{\Pi^{*}}$ is the identity element in $\Pi^{*}$.

(5) If $\tau_{1}>\tau_{2}$, then $\tilde{\delta}\left(\cdot, \tau_{1}\right)>\tilde{\delta}\left(\cdot, \tau_{2}\right)$.

A subset $S$ of $\mathbb{R}$ is called relatively dense with respect to the pair $\left(\Pi^{*}, \tilde{\delta}\right)$ if there exists a number $L \in \Pi^{*}$ and $L>e_{\Pi^{*}}$ such that $[a, \tilde{\delta}(a, L)]_{\Pi^{*}} \cap S \neq \emptyset$ for all $a \in \Pi^{*}$. The number $|L|$ is called the inclusion length with respect to the group $\left(\Pi^{*}, \tilde{\delta}\right)$.

Remark 2.1 Note that from Definition 2.1, one can derive some classical concepts of relatively dense set. Here, we present some classical concepts according to it.

(i) If $\Pi^{*}=\mathbb{R}$ and $\tilde{\delta}\left(\tau_{1}, \tau_{2}\right)=\tau_{1}+\tau_{2}$, then $e_{\Pi^{*}}=0$. One can obtain the following concept:

Definition 2.2 (Definition 1.10 from [1]) A subset $S$ of $\mathbb{R}$ is called relatively dense with respect to the pair $(\mathbb{R},+)$ if there exists a number $L>0$ such that $[a, a+L] \cap S \neq \emptyset$ for all $a \in \mathbb{R}$. The number $L$ is called the inclusion length with respect to the group $(\mathbb{R},+)$.

(ii) If $\Pi^{*}=h \mathbb{Z}, h>0$ and $\tilde{\delta}\left(\tau_{1}, \tau_{2}\right)=\tau_{1}+\tau_{2}$, then $e_{\Pi^{*}}=0$. We can immediately get the following concept:

Definition 2.3 A subset $S$ of $\mathbb{R}$ is called relatively dense with respect to the pair $(h \mathbb{Z},+)$ if there exists a number $L \in h \mathbb{Z}^{+}$such that $[a, a+L]_{h \mathbb{Z}} \cap S \neq \emptyset$ for all $a \in h \mathbb{Z}$. The number $L$ is called the inclusion length with respect to the group $(h \mathbb{Z},+)$.

(iii) If $\Pi^{*}=q^{\mathbb{Z}}:=\left\{q^{n}: q>1, n \in \mathbb{Z}\right\}$ and $\tilde{\delta}\left(\tau_{1}, \tau_{2}\right)=\tau_{1} \cdot \tau_{2}$, then $e_{\Pi^{*}}=1$. One can establish the following concept:

Definition 2.4 A subset $S$ of $\mathbb{R}$ is called relatively dense with respect to the pair $\left(q^{\mathbb{Z}}, \cdot\right)$ if there exists a number $L \in(1,+\infty)_{q^{\mathbb{Z}}}$ such that $[a, a L]_{q^{\mathbb{Z}}} \cap S \neq \emptyset$ for all $a \in q^{\mathbb{Z}}$. The number $L$ is called the inclusion length with respect to the group $\left(q^{\mathbb{Z}}, \cdot\right)$. 
(iv) If $\Pi^{*}=\mathbb{N}_{ \pm}^{\frac{1}{2}}:=\{ \pm \sqrt{n}, n \in \mathbb{N}\}$ and

$$
\tilde{\delta}\left(\tau_{1}, \tau_{2}\right)=\left\{\begin{array}{ll}
\sqrt{\tau_{1}^{2}+\operatorname{sgn}\left(\tau_{2}\right) \cdot \tau_{2}^{2}}, & \tau_{1}>0, \\
-\sqrt{\tau_{1}^{2}-\operatorname{sgn}\left(\tau_{2}\right) \cdot \tau_{2}^{2}}, & \tau_{1}<0,
\end{array} \quad \text { then } e_{\Pi^{*}}=0\right.
$$

One will have the following concept:

Definition 2.5 A subset $S$ of $\mathbb{R}$ is called relatively dense with respect to the pair $\left(\mathbb{N}_{ \pm}^{\frac{1}{2}}, \tilde{\delta}\right)$ if there exists a number $L \in(1,+\infty)_{\mathbb{N}_{+}^{\frac{1}{2}}}$ such that $\left[a, \sqrt{a^{2}+L^{2}}\right]_{\mathbb{N}_{+}^{\frac{1}{2}}} \cap S \neq \emptyset$ for all $a \in \mathbb{N}_{+}^{\frac{1}{2}}$ and $\left[a,-\sqrt{a^{2}-L^{2}}\right]_{\mathbb{N}^{\frac{1}{2}}} \cap S \neq \emptyset$ for all $a \in \mathbb{N}_{\underline{\frac{1}{2}}}$. The number $L$ is called the inclusion length with respect to the group $\left(\mathbb{N}_{ \pm}^{\frac{1}{2}}, \tilde{\delta}\right)$.

From Definitions 2.2-2.5, one will see that by employing Definition 2.1, some new concepts of relatively dense sets for various types of time scales can be derived. It is easy to observe that Definition 2.2 is suitable for $\mathbb{T}=\mathbb{R}$, Definition 2.3 is appropriate for $\mathbb{T}=h \mathbb{Z}$, Definition 2.4 is suitable for $\mathbb{T}=\overline{q^{\mathbb{Z}}}$ and Definition 2.5 is applicable to $\mathbb{T}=\mathbb{N}_{ \pm}^{\frac{1}{2}}$.

Similarly, by using the operator $\tilde{\delta}$, one can also establish the concepts of relatively dense sets for $\mathbb{T}=\overline{-q^{\mathbb{Z}} \cup q^{\mathbb{Z}}}$ and $\overline{(-q)^{\mathbb{Z}}}, q>1$, etc. Hence, it is obvious that Definition 2.1 is so general that it can unify the concepts of relatively dense sets for many irregular time scales, it will be a useful tool to describe the almost periodicity of functions on a more comprehensive scope of time scales.

According to Definition 2.1, one can obtain the following property.

Theorem 2.2 Let a pair $\left(\Pi^{*}, \tilde{\delta}\right)$ be an Abelian group and $\tau_{1}, \tau_{2} \in \Pi^{*}$. Then

$$
\tilde{\delta}^{-1}\left(\tau_{1}, \tau_{2}\right)=\tilde{\delta}\left(\tau_{1}^{-1}, \tau_{2}^{-1}\right)
$$

Proof Note the following:

$$
\begin{aligned}
\tilde{\delta}\left(\tilde{\delta}\left(\tau_{1}, \tau_{2}\right), \tilde{\delta}\left(\tau_{1}^{-1}, \tau_{2}^{-1}\right)\right) & =\tilde{\delta}\left(\tau_{1}, \tilde{\delta}\left(\tau_{2}, \tilde{\delta}\left(\tau_{1}^{-1}, \tau_{2}^{-1}\right)\right)\right) \\
& =\tilde{\delta}\left(\tau_{1}, \tilde{\delta}\left(\tilde{\delta}\left(\tau_{2}, \tau_{2}^{-1}\right), \tau_{1}^{-1}\right)\right) \\
& =\tilde{\delta}\left(\tau_{1}, \tilde{\delta}\left(e_{\Pi^{*}}, \tau_{1}^{-1}\right)\right) \\
& =\tilde{\delta}\left(\tau_{1}, \tau_{1}^{-1}\right)=e_{\Pi^{*}}
\end{aligned}
$$

Hence, we can obtain the desired result. This completes the proof.

Next, we introduce the concept of an adjoint mapping between $\mathbb{T}$ and $\Pi$ to lay the foundation for introducing matched spaces.

Definition 2.6 Let $\mathbb{T}$ and $\Pi$ be time scales, where $\mathbb{T}=\bigcup_{i \in I_{1}} A_{i}, \Pi=\bigcup_{i \in I_{2}} B_{i}$. If $\Pi^{*}$ is the largest subset of the time scale $\Pi$, i.e., $\overline{\Pi^{*}}=\Pi$, where $\bar{A}$ denotes the closure of the set $A$, and $\left(\Pi^{*}, \tilde{\delta}\right)$ is an Abelian group, $I_{1}, I_{2}$ are countable index sets, then we say $\Pi$ is an adjoint 
set of $\mathbb{T}$ if there exists a bijective mapping

$$
\begin{aligned}
F: \quad \mathbb{T} & \rightarrow \quad \Pi, \\
A \in\left\{A_{i}, i \in I_{1}\right\} & \rightarrow B \in\left\{B_{i}, i \in I_{2}\right\},
\end{aligned}
$$

i.e., $F(A)=B$. Now $F$ is called the adjoint mapping between $\mathbb{T}$ and $\Pi$.

Example 2.3 In the following, according to Definition 2.6, one can see that $\Pi$ is an adjoint set of $\mathbb{T}$.

(1) Consider the following time scale $\mathbb{T}$ and the set $\Pi$ :

$$
\mathbb{T}=\overline{\left\{2^{i}: i \in \mathbb{Z}\right\}}, \quad \Pi=\overline{\left\{3^{i}: i \in \mathbb{Z}\right\}},
$$

and obviously, there exists a bijective map

$$
\begin{aligned}
F: \quad & \rightarrow \frac{\Pi,}{\mathbb{T}} \\
A \in\left\{2^{i}, i \in \mathbb{Z}\right\} & \rightarrow B \in\left\{3^{i, i \in \mathbb{Z}\}},\right. \\
A & \rightarrow \quad A^{\log _{2} 3}=B, \\
A=0 & \rightarrow \quad B=0,
\end{aligned}
$$

i.e., $F(A)=B$. Note that $\Pi \backslash\{0\}=\Pi^{*} \not \subset \mathbb{T}$ and $e_{\Pi^{*}}=1$. Here, $\tilde{\delta}\left(\tau_{1}, \tau_{2}\right)=\tau_{1} \cdot \tau_{2}$.

(2) Consider the following time scale $\mathbb{T}$ and the set $\Pi$ :

$$
\mathbb{T}=\bigcup_{i \in \mathbb{Z}}[i(a+b), i(a+b)+b], \quad \text { where } a \neq-b, \Pi=\{i(a+b): i \in \mathbb{Z}\},
$$

and obviously, there exists a bijective mapping

$$
\begin{aligned}
& \begin{array}{lll}
F: & \mathbb{T} & \rightarrow
\end{array} \\
& A \in\{[i(a+b), i(a+b)+b], i \in \mathbb{Z}\} \rightarrow \quad B \in\{i(a+b), i \in \mathbb{Z}\}, \\
& A \quad \rightarrow \text { the left point of the interval } A \text {, }
\end{aligned}
$$

i.e., $F(A)=B$. Note that $\Pi=\Pi^{*} \subset \mathbb{T}$ and $e_{\Pi^{*}}=0$. Here, $\tilde{\delta}\left(\tau_{1}, \tau_{2}\right)=\tau_{1}+\tau_{2}$.

(3) Consider the following time scale $\mathbb{T}$ and the set $\Pi$ :

$$
\mathbb{T}=\bigcup_{i \in \mathbb{Z}}[i(a+b), i(a+b)+b], \quad \text { where } a>-b, \quad \Pi=\left\{ \pm \frac{i^{2}(a+b)}{\sqrt{3}}: i \in \mathbb{Z}\right\}
$$

and obviously, there exists a bijective mapping

$$
\begin{array}{ccc}
\mathbb{T}: & \rightarrow & \tilde{\Pi}_{1}, \\
A \in\{[i(a+b), i(a+b)+b], i \in \mathbb{Z}\} & \rightarrow \quad B_{1} \in\{i(a+b): i \in \mathbb{Z}\}, \\
A & \rightarrow \text { the left point of the interval } A,
\end{array}
$$

i.e., $\mathbb{Z}(A)=B_{1}$. Then

$$
\begin{aligned}
& \mathcal{N}: \quad \tilde{\Pi}_{1} \quad \rightarrow \quad \Pi \text {, } \\
& B_{1} \in\{i(a+b), i \in \mathbb{Z}\} \rightarrow B \in\left\{ \pm \frac{i^{2}(a+b)}{\sqrt{3}}: i \in \mathbb{Z}\right\} \text {, } \\
& B_{1} \quad \rightarrow \quad \frac{\operatorname{sgn}\left(B_{1}\right) B_{1}^{2}}{\sqrt{3}(a+b)}=B,
\end{aligned}
$$




$$
\begin{aligned}
& \text { i.e., } F(A)=\mathcal{N} \circ \mathcal{Z}(A)=B \text {. Note that } \Pi=\Pi^{*} \not \subset \mathbb{T} \text { and } e_{\Pi^{*}}=0 \text {. Here, } \\
& \qquad \tilde{\delta}\left(\tau_{1}, \tau_{2}\right)=\operatorname{sgn}\left(\operatorname{sgn}\left(\tau_{1}\right) \sqrt{\left|\tau_{1}\right|}+\operatorname{sgn}\left(\tau_{2}\right) \sqrt{\left|\tau_{2}\right|}\right)\left(\operatorname{sgn}\left(\tau_{1}\right) \sqrt{\left|\tau_{1}\right|}+\operatorname{sgn}\left(\tau_{2}\right) \sqrt{\left|\tau_{2}\right|}\right)^{2} .
\end{aligned}
$$

We introduce an operator $\delta$ with the following algebraic structure between $\mathbb{T}$ and $\Pi$.

Definition 2.7 Let the pair $\left(\Pi^{*}, \tilde{\delta}\right)$ be an Abelian group and $\Pi^{*}, \mathbb{T}^{*}$ be the largest subsets of the time scales $\Pi$ and $\mathbb{T}$, respectively. Further, let $\Pi$ be the adjoint set of $\mathbb{T}$ and $F$ be the adjoint mapping between $\mathbb{T}$ and $\Pi$. The operator $\delta: \Pi^{*} \times \mathbb{T}^{*} \rightarrow \mathbb{T}^{*}$ satisfies the following properties:

$\left(P_{1}\right)$ (Monotonicity) The function $\delta$ is strictly increasing with respect to its all arguments, i.e., if

$$
\left(T_{0}, t\right),\left(T_{0}, u\right) \in \mathcal{D}_{\delta}:=\left\{(s, t) \in \Pi^{*} \times \mathbb{T}^{*}: \delta(s, t) \in \mathbb{T}^{*}\right\},
$$

then $t<u$ implies $\delta\left(T_{0}, t\right)<\delta\left(T_{0}, u\right)$; if $\left(T_{1}, u\right),\left(T_{2}, u\right) \in \mathcal{D}_{\delta}$ with $T_{1}<T_{2}$, then $\delta\left(T_{1}, u\right)<$ $\delta\left(T_{2}, u\right)$.

$\left(P_{2}\right)$ (Existence of inverse elements) The operator $\delta$ has the inverse operator $\delta^{-1}: \Pi^{*} \times$ $\mathbb{T}^{*} \rightarrow \mathbb{T}^{*}$ and $\delta^{-1}(\tau, t)=\delta\left(\tau^{-1}, t\right)$, where $\tau^{-1} \in \Pi^{*}$ is the inverse element of $\tau$.

$\left(P_{3}\right)$ (Existence of identity element) $e_{\Pi^{*}} \in \Pi^{*}$ and $\delta\left(e_{\Pi^{*}}, t\right)=t$ for any $t \in \mathbb{T}^{*}$, where $e_{\Pi^{*}}$ is the identity element in $\Pi^{*}$.

$\left(P_{4}\right)$ (Bridge condition) For any $\tau_{1}, \tau_{2} \in \Pi^{*}$ and $t \in \mathbb{T}^{*}, \delta\left(\tilde{\delta}\left(\tau_{1}, \tau_{2}\right), t\right)=\delta\left(\tau_{1}, \delta\left(\tau_{2}, t\right)\right)=$ $\delta\left(\tau_{2}, \delta\left(\tau_{1}, t\right)\right)$.

Then the operator $\delta(s, t)$ associated with $e_{\Pi^{*}} \in \Pi^{*}$ is said to be a shift operator on the set $\mathbb{T}^{*}$. The variable $s \in \Pi^{*}$ in $\delta$ is called the shift size. The value $\delta(s, t)$ in $\mathbb{T}^{*}$ indicates $s$ units shift of the term $t \in \mathbb{T}^{*}$. The set $\mathcal{D}_{\delta}$ is the domain of the shift operator $\delta$.

Example 2.4 According to Definition 2.7, we provide a matching among $\Pi^{*}, \tilde{\delta}$ and $\delta$ for the following time scales.

(1) Let $\mathbb{T}=-\overline{q^{\mathbb{Z}}}=\overline{\left\{-q^{n}: q>1, n \in \mathbb{Z}\right\}}$. For such a time scale, take $e_{\Pi^{*}}=1$, we attach the shift operators

$$
\begin{aligned}
& \delta(s, t)=\frac{t}{s}, \\
& \tilde{\delta}\left(s_{1}, s_{2}\right)=s_{1} \cdot s_{2}
\end{aligned}
$$

and $\Pi^{*}=\left\{q^{n}: q>1, n \in \mathbb{Z}\right\}$.

(2) Let $\mathbb{T}=\overline{q^{\mathbb{Z}}}=\left\{q^{n}: q>1, n \in \mathbb{Z}\right\} \cup\{0\}$. For this time scale, take $e_{\Pi^{*}}=1$, we attach the shift operators

$$
\begin{aligned}
& \delta(s, t)=s t, \\
& \tilde{\delta}\left(s_{1}, s_{2}\right)=s_{1} \cdot s_{2}
\end{aligned}
$$

$$
\text { and } \Pi^{*}=\left\{q^{n}: q>1, n \in \mathbb{Z}\right\}
$$


(3) Let $\mathbb{T}=\overline{(-q)^{\mathbb{Z}}}=\left\{(-q)^{n}: q>1, n \in \mathbb{Z}\right\} \cup\{0\}$. For such a time scale and any $t \in \mathbb{T}^{*}$, take $e_{\Pi^{*}}=1$, we attach the shift operators

$$
\delta(s, t)=\left\{\begin{array}{ll}
s t, & t>0, \\
\frac{t}{s}, & t<0,
\end{array} \quad \tilde{\delta}\left(s_{1}, s_{2}\right)=s_{1} \cdot s_{2}\right.
$$

and $\Pi^{*}=\left\{(-q)^{2 n}: q>1, n \in \mathbb{Z}\right\}$.

(4) Consider $\mathbb{T}=\overline{-q^{\mathbb{Z}} \cup q^{\mathbb{Z}}}=\left\{q^{n}: q>1, n \in \mathbb{Z}\right\} \cup\left\{-q^{n}: q>1, n \in \mathbb{Z}\right\} \cup\{0\}$. For this time scale and any $t \in \mathbb{T}^{*}$, take $e_{\Pi^{*}}=1$, we attach the shift operators

$$
\delta(s, t)=\left\{\begin{array}{ll}
s t, & t>0, \\
\frac{t}{s}, & t<0,
\end{array} \quad \tilde{\delta}\left(s_{1}, s_{2}\right)=s_{1} \cdot s_{2}\right.
$$

and $\Pi^{*}=\left\{q^{n}: q>1, n \in \mathbb{Z}\right\}$

(5) Consider $\mathbb{N}_{ \pm}^{\frac{1}{2}}=\{ \pm \sqrt{n}, n \in \mathbb{N}\}$. For any $t \in \mathbb{T}^{*}$, take $e_{\Pi^{*}}=0$, we attach the shift operators

$$
\delta(s, t)=\left\{\begin{array}{ll}
\sqrt{t^{2}+\operatorname{sgn}(s) \cdot s^{2}}, & t>0, \\
-\sqrt{t^{2}-\operatorname{sgn}(s) \cdot s^{2}}, & t<0,
\end{array} \quad \tilde{\delta}\left(s_{1}, s_{2}\right)= \begin{cases}\sqrt{s_{1}^{2}+\operatorname{sgn}\left(s_{2}\right) \cdot s_{2}^{2}}, & s_{1}>0 \\
-\sqrt{s_{1}^{2}-\operatorname{sgn}\left(s_{2}\right) \cdot s_{2}^{2}}, & s_{1}<0\end{cases}\right.
$$

and $\Pi^{*}=\mathbb{N}_{ \pm}^{\frac{1}{2}}=\{ \pm \sqrt{n}: n \in \mathbb{N}\}$

In the next example, one will see that the condition $\left(P_{2}\right)$ from Definition 2.7 may not be satisfied but the complete closedness for some types of time scales still can be guaranteed under the shift $\delta$.

Example 2.5 Let $\mathbb{T}_{1}=\overline{\left\{q^{n}: q>1, n \in \mathbb{Z}^{+}\right\}}$and $\mathbb{T}_{2}=\overline{\left\{q^{n}: q>1, n \in \mathbb{Z}^{-}\right.}$. For these two time scales, $\Pi_{1}=\mathbb{T}_{1}, \Pi_{2}=\mathbb{T}_{2}$. Denote the adjoint mapping between $\mathbb{T}_{1}$ and $\Pi_{1}$ by $F_{1}$, and the mapping between $\mathbb{T}_{2}$ and $\Pi_{2}$ by $F_{2}$, then it is easy to obtain $F_{1}=F_{2}=I$, where $I$ is an identity mapping. Take $e_{\Pi^{*}}=1$ and $\Pi^{*}=\left\{q^{n}: q>1, n \in \mathbb{Z}\right\}=\Pi_{1}^{*} \cup \Pi_{2}^{*}$, where $\Pi_{1}^{*}=\left\{q^{n}: q>\right.$ $\left.1, n \in \mathbb{Z}^{+}\right\} \cup\{1\}, \Pi_{2}^{*}=\left\{q^{n}: q>1, n \in \mathbb{Z}^{-}\right\} \cup\{1\}, \tilde{\delta}_{1}=\tilde{\delta}_{2}=s_{1} s_{2}$.

It is clear that for any $s_{1} \in \Pi_{1}^{*}, s_{2} \in \Pi_{2}^{*}$, we obtain $\delta_{1}\left(s_{1}, t_{1}\right)=s_{1} t_{1} \in \mathbb{T}_{1}^{*}$ for all $t_{1} \in \mathbb{T}_{1}^{*}$ but $s_{1}^{-1} \notin \Pi_{1}^{*} \backslash\{1\}$, which implies that $\delta_{1}\left(s_{1}^{-1}, t_{1}\right)=\frac{t_{1}}{s_{1}} \notin \mathbb{T}_{1}^{*}$ for $t_{1}=q, s_{1}=q^{2}$.

Similarly, $\delta_{2}\left(s_{2}, t_{2}\right)=s_{2} t_{2} \in \mathbb{T}_{2}^{*}$ for all $t_{2} \in \mathbb{T}_{2}^{*}$ but $s_{2}^{-1} \notin \Pi_{2}^{*} \backslash\{1\}$, which implies that $\delta_{2}\left(s_{2}^{-1}, t_{2}\right)=\frac{t_{2}}{s_{2}} \notin \mathbb{T}_{2}^{*}$ for $t_{2}=\frac{1}{q}, s_{2}=\frac{1}{q^{2}}$.

Hence, under the shift operators $\delta_{1}(s, t)=\delta_{2}(s, t)=s t$, one can see that $\mathbb{T}_{1}$ and $\mathbb{T}_{2}$ are completely closed. However, all the elements from $\Pi_{1}^{*} \backslash\{1\}$ and $\Pi_{2}^{*} \backslash\{1\}$ have no corresponding inverse elements in $\Pi_{1}^{*}$ and $\Pi_{2}^{*}$, respectively, that is, $\left(\Pi_{1}^{*}, \tilde{\delta}_{1}\right)$ and $\left(\Pi_{2}^{*}, \tilde{\delta}_{2}\right)$ are not Abelian groups but they can guarantee the complete closedness of time scales $\mathbb{T}_{1}$ and $\mathbb{T}_{2}$.

Remark 2.6 From the condition $\left(P_{2}\right)$ in Definition 2.7, one can easily observe that $\delta^{-1}$ exists if and only if $\left(\Pi^{*}, \tilde{\delta}\right)$ is an Abelian group.

In order to include the cases of the closedness of time scales from Example 2.5, it is significant to propose the following concept of shift operator $\delta$ attached with shift directions. 
Definition 2.8 Let the pair $\left(\Pi^{*}, \tilde{\delta}\right)$ be closed towards the operation $\tilde{\delta}$, and $\Pi^{*}, \mathbb{T}^{*}$ be the largest subsets of the time scales $\Pi$ and $\mathbb{T}$, respectively. Further, let $\Pi$ be the adjoint set of $\mathbb{T}$ and $F$ the adjoint mapping between $\mathbb{T}$ and $\Pi$. The operator $\delta: \Pi^{*} \times \mathbb{T}^{*} \rightarrow \mathbb{T}^{*}$ satisfies the following properties:

$\left(P_{1}\right)$ (Monotonicity) The function $\delta$ is strictly increasing with respect to all its arguments, i.e., if

$$
\left(T_{0}, t\right),\left(T_{0}, u\right) \in \mathcal{D}_{\delta}:=\left\{(s, t) \in \Pi^{*} \times \mathbb{T}^{*}: \delta(s, t) \in \mathbb{T}^{*}\right\}
$$

then $t<u$ implies $\delta\left(T_{0}, t\right)<\delta\left(T_{0}, u\right)$; if $\left(T_{1}, u\right),\left(T_{2}, u\right) \in \mathcal{D}_{\delta}$ with $T_{1}<T_{2}$, then $\delta\left(T_{1}, u\right)<$ $\delta\left(T_{2}, u\right)$.

$\left(P_{2}\right)$ (Existence of identity element) $e_{\Pi^{*}} \in \Pi^{*}$ and $\delta\left(e_{\Pi^{*}}, t\right)=t$ for any $t \in \mathbb{T}^{*}$, where $e_{\Pi^{*}}$ is the identity element in $\Pi^{*}$.

$\left(P_{3}\right)$ (Bridge condition) For any $\tau_{1}, \tau_{2} \in \Pi^{*}$ and $t \in \mathbb{T}^{*}, \delta\left(\tilde{\delta}\left(\tau_{1}, \tau_{2}\right), t\right)=\delta\left(\tau_{1}, \delta\left(\tau_{2}, t\right)\right)=$ $\delta\left(\tau_{2}, \delta\left(\tau_{1}, t\right)\right)$.

Then the operator $\delta$ associated with $e_{\Pi^{*}} \in \Pi^{*}$ is said to be shift operator on the set $\mathbb{T}^{*}$. The variable $s \in \Pi^{*}$ in $\delta$ is called the shift size. The value $\delta(s, t)$ in $\mathbb{T}^{*}$ indicates $s$ units shift of the term $t \in \mathbb{T}^{*}$. The set $\mathcal{D}_{\delta}$ is the domain of the shift operator $\delta$.

Remark 2.7 Note that the condition $\left(P_{2}\right)$ from Definition 2.7 is not satisfied in Definition 2.8 since $\left(\Pi^{*}, \tilde{\delta}\right)$ may not be an Abelian group in Definition 2.8, i.e., $\delta^{-1}$ may not exist.

Definition 2.9 Under Definition 2.8, we introduce three types of shift operators:

(1) We say $\delta$ is a positive-direction shift operator if for any $p>e_{\Pi^{*}}$ and $p \in \Pi^{*}$, there exists a number $P>p$ and $P \in \Pi^{*}$ such that $\delta(P, t) \in \mathbb{T}^{*}$ for all $t \in \mathbb{T}^{*}$.

(2) We say $\delta$ is a negative-direction shift operator if for any $q<e_{\Pi^{*}}$ and $q \in \Pi^{*}$, there exists a number $Q<q$ and $Q \in \Pi^{*}$ such that $\delta(Q, t) \in \mathbb{T}^{*}$ for all $t \in \mathbb{T}^{*}$.

(3) We say $\delta$ is a bi-direction shift operator if for any $p>e_{\Pi^{*}}$ and $q<e_{\Pi^{*}}$, where $p, q \in \Pi^{*}$, there exist two numbers $P>p, Q<q$ and $P, Q \in \Pi^{*}$ such that $\delta(P, t), \delta(Q, t) \in \mathbb{T}^{*}$ for all $t \in \mathbb{T}^{*}$.

(4) We say $\delta$ is an oriented-direction shift operator if $\delta$ is a positive-direction shift operator or a negative-direction shift operator.

Remark 2.8 Under Definition 2.9, the complete closedness of time scales in Example 2.5 can be well described, that is, $\delta$ for $\mathbb{T}_{1}$ is a positive-direction shift operator, $\delta$ for $\mathbb{T}_{2}$ is a negative-direction shift operator. In addition, all the operators $\delta$ in Example 2.4 are bidirection shift operators. Also, one can observe that the sets $\left(\Pi^{*}, \tilde{\delta}\right)$ in Example 2.4 are Abelian groups, but $\left(\Pi_{1}^{*}, \tilde{\delta}_{1}\right)$ and $\left(\Pi_{2}^{*}, \tilde{\delta}_{2}\right)$ in Example 2.5 are not Abelian groups.

Remark 2.9 According to Definition 2.9, we can derive the following related concepts, which indicates that the shifts of time scales under the operator $\delta$ include the cases of the translations of time scales.

(i) If $\mathbb{T}=\mathbb{R}$, then $\delta(s, t)=t+s$, where $s \in \Pi^{*}=\mathbb{R}$ and $\tilde{\delta}\left(s_{1}, s_{2}\right)=s_{1}+s_{2}$. It is easy to observe that $\left(\Pi^{*}, \tilde{\delta}\right)$ forms an Abelian group, so one can easily obtain that $\delta$ is a bi-direction shift operator. 
(ii) If $\mathbb{T}=h \mathbb{Z}, h>0$, then $\delta(s, t)=t+s$, where $s \in \Pi^{*}=h \mathbb{Z}$ and $\tilde{\delta}\left(s_{1}, s_{2}\right)=s_{1}+s_{2}$. Also, one can easily get that $\delta$ is a bi-direction shift operator because $\left(\Pi^{*}, \tilde{\delta}\right)$ forms an Abelian group.

(iii) If $\mathbb{T}=\bigcup_{k=0}^{+\infty}[2 k, 2 k+1]$, then $\delta(s, t)=t+s$, where $s \in \Pi^{*}=\{2 n, n \in \mathbb{N}\}$ and $\tilde{\delta}\left(s_{1}, s_{2}\right)=s_{1}+s_{2}$. Since each element from $\Pi^{*}$ has no inverse element in $\Pi^{*} \backslash\{0\}$ with respect to the operator $\tilde{\delta}$, i.e., $\left(\Pi^{*}, \tilde{\delta}\right)$ is not an Abelian group. In fact, one can easily see that $\delta$ is a positive-direction shift operator.

In the literature [17], the authors proposed the concept of time scales attached with translation direction to discuss the closedness of time scales under translations. In this paper, one can observe that the translation of time scales is just a particular case of the shift of time scales. Hence, it is significant to consider the closedness of time scales under shifts because not only can it unify the discrete and continuous time scales (i.e., $\mathbb{T}=h \mathbb{Z}$ or $\mathbb{R}$ ) but also the quantum time scale (i.e., $\mathbb{T}=\overline{q^{\mathbb{Z}}}$ ) and other irregular types of time scales such as $\overline{(-q)^{\mathbb{Z}}}, \mathbb{N}_{ \pm}^{\frac{1}{2}}$, etc.

For convenience, we introduce the following concepts of semigroup of $\Pi^{*}$ attached with shift direction.

Definition 2.10 Let $\left(\Pi^{*}, \delta\right)$ be an Abelian group and

$$
\Pi_{1}^{* *}:=\left\{\tau \in \Pi^{*}: s \leq e_{\Pi^{*}}\right\}, \quad \Pi_{2}^{* *}:=\left\{\tau \in \Pi^{*}: s \geq e_{\Pi^{*}}\right\}
$$

Then the sets $\Pi_{1}^{* *}$ and $\Pi_{2}^{* *}$ are called the negative-direction semigroup and the positivedirection semigroup for $\Pi^{*}$, respectively.

According to Definition 2.10, the following theorem is obvious.

Theorem 2.10 If $\Pi^{*}$ is a semigroup with negative-direction (or positive-direction), then $\delta$ is a negative-direction shift operator (or a positive-direction shift operator).

In what follows, we assume that $\delta$ is a bi-direction shift operator, i.e., $\left(\Pi^{*}, \tilde{\delta}\right)$ forms an Abelian group. From Definition 2.7, we introduce the concept of matched spaces for time scales and establish some related properties.

Definition 2.11 Let the pair $\left(\Pi^{*}, \tilde{\delta}\right)$ be an Abelian group and $\Pi^{*}, \mathbb{T}^{*}$ be the largest subsets of the time scales $\Pi$ and $\mathbb{T}$, respectively. Further, let $\Pi$ be an adjoint set of $\mathbb{T}$ and $F$ be the adjoint mapping between $\mathbb{T}$ and $\Pi$. If there exists the shift operator $\delta$ satisfying Definition 2.7 , then we say the group $(\mathbb{T}, \Pi, F, \delta)$ is a matched space for the time scale $\mathbb{T}$.

Remark 2.11 Note that Definition 2.11 reflects the algebraic structure of matched spaces, i.e, a matched space for the time scale is the group $(\mathbb{T}, \Pi, F, \delta)$.

Definition 2.12 If $\mathbb{T}=\Pi$, then we say the group $(\mathbb{T}, \Pi, F, \delta)$ is a standard matched space.

Remark 2.12 It is easy to see that for a standard matched space, the adjoint mapping $F$ is a unit operator $I$, for simplicity, $(\mathbb{T}, \Pi, F, \delta):=(\mathbb{T}, \delta)$. Under the standard matched space $(\mathbb{T}, \delta)$, one can employ the algebraic structure to include all the results from [15]. In fact, let 
$\Pi=\mathbb{T}, e_{\Pi^{*}}=t_{0}, \delta(s, t)=\delta_{+}(s, t)$ and $\delta\left(s^{-1}, t\right)=\delta^{-1}(s, t)=\delta_{-}(s, t)$, where $s \in\left[t_{0},+\infty\right)_{\mathbb{T}^{*}}$ and $t_{0}$ is an initial point, then all the results from [15] become a particular case of the established results in this paper.

Lemma 2.13 Let $\delta$ be a shift operator under Definition 2.7. Then the following are fulfilled:

(1) $\delta^{-1}\left(e_{\Pi^{*}}, t\right)=\delta\left(e_{\Pi^{*}}, t\right)=t$ for all $t \in \mathbb{T}^{*}$.

(2) If $(s, u) \in \mathcal{D}_{\delta^{-1}}$ and $(s, t) \in \mathcal{D}_{\delta}$, then $\delta(s, t)=u$ implies $\delta^{-1}(s, u)=t$ and $\delta^{-1}(s, u)=t$ implies $\delta(s, t)=u$.

(3) If $\left(T_{1}, u\right),\left(T_{2}, u\right) \in \mathcal{D}_{\delta^{-1}}$ and $T_{1} T_{2}>0, T_{1}<T_{2}$, then $\delta^{-1}\left(T_{1}, u\right)>\delta^{-1}\left(T_{2}, u\right)$.

(4) $\delta\left(\tau, \delta^{-1}\left(s, t_{0}\right)\right)=\delta^{-1}\left(s, \delta\left(\tau, t_{0}\right)\right)$ for any $t_{0} \in \mathbb{T}^{*}$.

(5) $\delta\left(\tilde{\delta}(u, s), \delta^{-1}(s, v)\right)=\delta(u, v) ; \delta^{-1}(\tilde{\delta}(u, s), \delta(s, v))=\delta^{-1}(u, v)$.

(6) If $\delta(\tau, \cdot)$ is $\Delta$-differentiable in its second variable, then $\delta^{\Delta}(\tau, \cdot)>0$.

Proof (1) Since $\delta^{-1}\left(e_{\Pi^{*}}, t\right)=\delta\left(e_{\Pi^{*}}^{-1}, t\right)=\delta\left(e_{\Pi^{*}}, t\right)$ for all $t \in \mathbb{T}^{*}$, we can obtain the result.

(2) If $(s, u) \in \mathcal{D}_{\delta^{-1}}$ and $(s, t) \in \mathcal{D}_{\delta}$, then

$$
\delta^{-1}(s, u)=\delta\left(s^{-1}, u\right)=\delta\left(s^{-1}, \delta(s, t)\right)=\delta\left(\tilde{\delta}\left(s^{-1}, s\right), t\right)=\delta\left(e_{\Pi^{*}}, t\right)=t
$$

and

$$
\delta(s, t)=\delta\left(s, \delta^{-1}(s, u)\right)=\delta\left(s, \delta\left(s^{-1}, u\right)\right)=\delta\left(\tilde{\delta}\left(s, s^{-1}\right), u\right)=\delta\left(e_{\Pi^{*}}, u\right)=u .
$$

(3) Since $T_{1}^{-1}>T_{2}^{-1}$, it follows from $\left(P_{1}\right),\left(P_{2}\right)$ in Definition 2.7 that

$$
\delta^{-1}\left(T_{1}, u\right)=\delta\left(T_{1}^{-1}, u\right)>\delta\left(T_{2}^{-1}, u\right)=\delta^{-1}\left(T_{2}, u\right)
$$

(4) We can directly calculate that

$$
\delta\left(\tau, \delta\left(s^{-1}, t_{0}\right)\right)=\delta\left(\tilde{\delta}\left(\tau, s^{-1}\right), t_{0}\right)=\delta\left(\tilde{\delta}\left(s^{-1}, \tau\right), t_{0}\right)=\delta\left(s^{-1}, \delta\left(\tau, t_{0}\right)\right)=\delta^{-1}\left(s, \delta\left(\tau, t_{0}\right)\right)
$$

(5) Through calculation, we obtain

$$
\begin{aligned}
\delta\left(\tilde{\delta}(u, s), \delta^{-1}(s, v)\right) & =\delta\left(u, \delta\left(s, \delta\left(s^{-1}, v\right)\right)\right)=\delta\left(u, \delta\left(\tilde{\delta}\left(s, s^{-1}\right), v\right)\right) \\
& =\delta\left(u, \delta\left(e_{\Pi^{*}}, v\right)\right)=\delta(u, v)
\end{aligned}
$$

and

$$
\begin{aligned}
\delta^{-1}(\tilde{\delta}(u, s), \delta(s, v)) & =\delta\left(\tilde{\delta}^{-1}(u, s), \delta(s, v)\right) \\
& =\delta\left(\tilde{\delta}\left(u^{-1}, s^{-1}\right), \delta(s, v)\right)=\delta\left(u^{-1}, \delta\left(s^{-1}, \delta(s, v)\right)\right) \\
& =\delta\left(u^{-1}, \delta\left(e_{\Pi^{*}}, v\right)\right)=\delta\left(u^{-1}, v\right)=\delta^{-1}(u, v) .
\end{aligned}
$$

(6) Since $\delta(\tau, \cdot)$ is strictly increasing in its second variable, we have (6) by Corollary 1.16 from [8]. This completes the proof.

Corollary 2.14 Let $\mathbb{T}^{*}$ be the largest subset of the time scale $\mathbb{T}$. For a standard matched space $(\mathbb{T}, \delta), \delta(\tau, t)=\delta(t, \tau)$ holds for all $(\tau, t) \in \mathbb{T}^{*} \times \mathbb{T}^{*}$. 
Proof Note that we obtain

$$
\begin{aligned}
\delta(\tau, t) & =\delta\left(e_{\Pi}, \delta(\tau, t)\right)=\delta\left(\tilde{\delta}\left(t, t^{-1}\right), \delta(\tau, t)\right)=\delta\left(t, \delta\left(t^{-1}, \delta(\tau, t)\right)\right) \\
& =\delta\left(t, \delta\left(\tilde{\delta}\left(t^{-1}, \tau\right), t\right)\right)=\delta\left(t, \delta\left(\tilde{\delta}\left(\tau, t^{-1}\right), t\right)\right)=\delta\left(t, \delta\left(\tau, \delta\left(t^{-1}, t\right)\right)\right) \\
& =\delta\left(t, \delta\left(\tau, e_{\Pi^{*}}\right)\right)=\delta(t, \tau)
\end{aligned}
$$

This completes the proof.

Next, we provide several examples to show the algebraic structure of matched spaces for time scales.

\section{Example 2.15}

(1) $\mathbb{T}=\left\{ \pm n^{2}: n \in \mathbb{Z}\right\}, \Pi=\{\tau: \tau=n, n \in \mathbb{Z}\}$, thus, the shift operator $\delta$ can be taken as follows:

$$
\delta(\tau, t)=\left\{\begin{array}{ll}
\operatorname{sgn}(\sqrt{t}+\tau) \cdot(\sqrt{t}+\tau)^{2}, & \text { if } t>0, \\
\operatorname{sgn}(\tau) \tau^{2}, & \text { if } t=0, \\
-\operatorname{sgn}(\sqrt{-t}-\tau) \cdot(\sqrt{-t}-\tau)^{2}, & \text { if } t<0 .
\end{array} \quad \tau=n_{0}, \quad \forall n_{0} \in \mathbb{Z} .\right.
$$

Thus, $\forall \tau_{1}, \tau_{2} \in \Pi^{*}=\Pi, \tilde{\delta}\left(\tau_{1}, \tau_{2}\right)=\tau_{1}+\tau_{2}$. Obviously, $\tilde{\delta}\left(\tau_{1}, \tau_{2}\right) \in \Pi^{*}$. On the other hand, there exists a bijective mapping

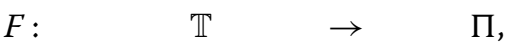

$$
\begin{aligned}
& A \in\left\{ \pm n^{2}, n \in \mathbb{Z}\right\} \rightarrow B \in\{n, n \in \mathbb{Z}\}, \\
& A \quad \rightarrow \operatorname{sgn}(A) \sqrt{|A|}=B,
\end{aligned}
$$

i.e., $F(A)=\operatorname{sgn}(A) \sqrt{|A|}$. Hence, we can obtain a matched space $(\mathbb{T}, \Pi, F, \delta)$ for the time scale $\mathbb{T}$.

(2) $\mathbb{T}=\overline{q^{\mathbb{Z}}}$ and $\Pi=\overline{q^{\mathbb{Z}}} . \delta(\tau, t)=\tau t, \tau=q^{n_{0}}, \forall n_{0} \in \mathbb{Z}$. Thus, $\forall \tau_{1}, \tau_{2} \in \Pi^{*}=\Pi \backslash\{0\}$, $\tilde{\delta}\left(\tau_{1}, \tau_{2}\right)=\tau_{1} \cdot \tau_{2}$. Obviously, $\tilde{\delta}\left(\tau_{1}, \tau_{2}\right) \in \Pi^{*}$. On the other hand, there exists a bijective mapping

$$
\begin{aligned}
F: \quad & \rightarrow \frac{\mathbb{T}}{A \in \overline{\left\{q^{n}, n \in \mathbb{Z}\right\}}} \rightarrow B \in\left\{q^{n}, n \in \mathbb{Z}\right\} \\
A & \rightarrow \quad A=B,
\end{aligned}
$$

i.e., $F(A)=A$. Hence, we can obtain a matched space $(\mathbb{T}, \Pi, F, \delta)$ for the time scale $\mathbb{T}$.

(3) $\mathbb{T}=\overline{\bigcup_{n \in \mathbb{Z}}\left[2^{2 n}, 2^{2 n+1}\right]}$ and $\Pi=\overline{\left\{\tau: \tau=2^{2 n}, n \in \mathbb{Z}\right\}} . \delta(\tau, t)=\tau t, \tau=2^{2 n_{0}}, \forall n_{0} \in \mathbb{Z}$.

Thus, $\forall \tau_{1}, \tau_{2} \in \Pi^{*}=\Pi \backslash\{0\}, \tilde{\delta}\left(\tau_{1}, \tau_{2}\right)=\tau_{1} \cdot \tau_{2}$. Obviously, $\tilde{\delta}\left(\tau_{1}, \tau_{2}\right) \in \Pi^{*}$. On the other hand, there exists a bijective mapping

$$
\begin{aligned}
& F: \quad \frac{\mathbb{T}}{A \in \overline{\bigcup_{n \in \mathbb{Z}}\left[2^{2 n}, 2^{2 n+1}\right]}} \rightarrow \quad B \in\left\{\frac{\Pi,}{\left\{\tau: \tau=2^{2 n}, n \in \mathbb{Z}\right\},}\right. \\
& A \quad \rightarrow \text { the left point of the interval } A \\
& A=0 \quad \rightarrow \quad B=0,
\end{aligned}
$$

i.e., $F(A)=B$. Hence, we can obtain a matched space $(\mathbb{T}, \Pi, F, \delta)$ for $\mathbb{T}$. 
(4) $\mathbb{T}=\left\{\frac{q^{n}}{1+q^{n}}: q>1\right.$ is a constant and $\left.n \in \mathbb{Z}\right\} \cup\{0,1\}$ and $\Pi=\overline{\left\{\tau: \tau=q^{n}, n \in \mathbb{Z}\right\}}$. Thus, the shift operators can be as follows:

$$
\delta(\tau, t)=\frac{q^{\frac{1}{\ln q}\left(\ln \frac{t}{1-t}+\ln \tau\right)}}{1+q^{\frac{1}{\ln q}\left(\ln \frac{t}{1-t}+\ln \tau\right)}}, \quad \tau=q^{n_{0}}, \forall n_{0} \in \mathbb{Z}
$$

Thus, we can obtain $\tilde{\delta}\left(\tau_{1}, \tau_{2}\right)=\tau_{1} \cdot \tau_{2}$. Obviously, $\tilde{\delta}\left(\tau_{1}, \tau_{2}\right) \in \Pi^{*}=\Pi \backslash\{0\}$. On the other hand, there exists a bijective mapping

$$
\begin{aligned}
& F: \quad \mathbb{T} \quad \rightarrow \quad \Pi \text {, } \\
& A \in\left\{\frac{q^{n}}{1+q^{n}}:\right. \\
& q>1 \text { is a constant and } n \in \mathbb{Z}\} \cup\{0,1\} \rightarrow B \in \overline{\left\{\tau: \tau=q^{n}, n \in \mathbb{Z}\right\}} \text {, } \\
& A \quad \rightarrow \quad \frac{A}{1-A}, \\
& A=0 \quad \rightarrow \quad B=0, \\
& A=1 \quad \rightarrow \quad B=1,
\end{aligned}
$$

i.e., $F(A)=B$. Hence, we can obtain a matched space $(\mathbb{T}, \Pi, F, \delta)$ for $\mathbb{T}$.

(5) If $\mathbb{T}=\bigcup_{k \in \mathbb{Z}}[k(a+b), k(a+b)+b]$, where $a \neq-b$ and $\Pi=\{\tau: \tau=n(a+b), n \in \mathbb{Z}\}$.

$\delta(\tau, t)=t+\tau, \tau=n_{0}(a+b), \forall n_{0} \in \mathbb{Z}$. Thus, we can obtain $\tilde{\delta}\left(\tau_{1}, \tau_{2}\right)=\tau_{1}+\tau_{2}$.

Obviously, $\tilde{\delta}\left(\tau_{1}, \tau_{2}\right) \in \Pi^{*}=\Pi$. On the other hand, there exists a bijective mapping

$$
\begin{aligned}
& F: \quad \mathbb{T} \quad \rightarrow \quad \Pi \text {, } \\
& A \in \bigcup_{k \in \mathbb{Z}}[k(a+b), k(a+b)+b] \rightarrow B \in\{\tau: \tau=n(a+b), n \in \mathbb{Z}\}, \\
& A \quad \rightarrow \text { the left point of the interval } A \text {, }
\end{aligned}
$$

i.e., $F(A)=B$. Hence, we can obtain a matched space $(\mathbb{T}, \Pi, F, \delta)$ for $\mathbb{T}$.

Using the algebraic structure of matched spaces, we introduce the following new concept of periodic time scales.

Definition 2.13 A time scale $\mathbb{T}$ is called a periodic time scale under a matched space $(\mathbb{T}, \Pi, F, \delta)$ if

$$
\tilde{\Pi}:=\left\{\tau \in \Pi^{*}:\left(\tau^{ \pm 1}, t\right) \in \mathcal{D}_{\delta}, \forall t \in \mathbb{T}^{*}\right\} \notin\left\{\left\{e_{\Pi^{*}}\right\}, \emptyset\right\} .
$$

Remark 2.16 From Definition 2.13, one should note that $\tilde{\Pi} \subseteq \Pi^{*} \subseteq \Pi$ and for every $\tau \in$ $\tilde{\Pi}$, there exists $\tau^{-1} \in \tilde{\Pi}$, i.e., there exists an inverse element for every element in $\tilde{\Pi}$.

Remark 2.17 For any $t \in \mathbb{T}^{*}$, if $\left(\tau_{2}, t\right) \in \mathcal{D}_{\delta},\left(\tau_{1}, t\right) \in \mathcal{D}_{\delta}$, then by Definition 2.7 , there exists a function $\tilde{\delta}: \Pi^{*} \times \Pi^{*} \rightarrow \Pi^{*}$ such that $\tilde{\delta}\left(\tau_{1}, \tau_{2}\right) \in \Pi^{*}$. Obviously, if $\mathbb{T}$ is a periodic time scale in the sense of Definition 1.1, i.e., $e_{\Pi^{*}}=0$ and $\tilde{\Pi}=\Pi^{*}=\Pi=\left\{\tau \in \mathbb{R}: t \pm \tau \in \mathcal{D}_{\delta}, \forall t \in\right.$ $\mathbb{T}\} \notin\{\{0\}, \emptyset\}$, we have $\tau_{1}, \tau_{2} \in \tilde{\Pi}$, then $\tilde{\delta}\left(\tau_{1}, \tau_{2}\right)=\tau_{1}+\tau_{2} \in \tilde{\Pi}$.

Remark 2.18 From (3), one can see $\left(\tau^{ \pm 1}, t\right) \in \mathcal{D}_{\delta}$ implies that $\delta\left(\tau^{-1}, t\right)=\delta^{-1}(\tau, t)$ exists.

In the following, we present some periodic time scales in the sense of Definition 2.13. 
Example 2.19 In Example 2.15, we can obtain the operators $\delta$ as follows:

(1)

$$
\delta(\tau, t)= \begin{cases}\operatorname{sgn}(\sqrt{t}+\tau) \cdot(\sqrt{t}+\tau)^{2}, & \text { if } t>0, \tau= \pm 1, \\ \operatorname{sgn}(\tau), & \text { if } t=0, \tau= \pm 1, \\ -\operatorname{sgn}(\sqrt{-t}-\tau) \cdot(\sqrt{-t}-\tau)^{2}, & \text { if } t<0, \tau= \pm 1 .\end{cases}
$$

(2) $\mathbb{T}=\overline{q^{\mathbb{Z}}}, \delta(\tau, t)=\tau t, \tau=q^{ \pm 1}$.

(3) $\mathbb{T}=\overline{\bigcup_{n \in \mathbb{Z}}\left[2^{2 n}, 2^{2 n+1}\right]}, \delta(\tau, t)=\tau t, \tau=4^{ \pm 1}$.

(4) $\delta(\tau, t)=\frac{q^{\frac{1}{\ln q}\left(\ln \frac{t}{1-t}+\ln \tau\right)}}{1+q^{\frac{1}{\ln q}\left(\ln \frac{t}{1-t}+\ln \tau\right)}}, \tau=q^{ \pm 1}$.

(5) $\delta(\tau, t)=t+\tau, \tau= \pm(a+b)$.

From the above and the operators $\delta$, one can obtain that the above numbers $\tau \in \tilde{\Pi}$. Hence, (1)-(5) are periodic time scales under their matched spaces. Furthermore, it is also easy to see that (1)-(4) from Example 2.15 are not periodic time scales in the sense of Definition 1.1.

Remark 2.20 We now show that (1), (3) in Example 2.3 are periodic under their matched spaces. However, they are not periodic in the sense of Definition 1.2. In fact, one can easily observe that $\mathbb{T} \cap \Pi^{*}=\emptyset$ in (1), (3) in Example 2.3, then Definition 1.2 is not satisfied since Definition 1.2 requires $\Pi^{*} \subset \mathbb{T}$. In fact, we have

(i) for (1) from Example 2.3, let $\delta(\tau, t)=t^{\log _{3} \tau}, \tau=3^{ \pm 1} \in \Pi^{*}$, and one can obtain $\delta(\tau, t) \in \mathbb{T}^{*}$ under the matched space $(\mathbb{T}, \Pi, F, \delta)$.

(ii) for (3) from Example 2.3, let

$$
\delta(\tau, t)=t+\operatorname{sgn}(\tau) \sqrt{\sqrt{3}(a+b)|\tau|}, \quad \tau= \pm \frac{a+b}{\sqrt{3}} \in \Pi^{*},
$$

and one can obtain $\delta(\tau, t) \in \mathbb{T}^{*}$ under the matched space $(\mathbb{T}, \Pi, F, \delta)$.

In what follows, denote the shift by $\delta_{\tau}(t):=\delta(\tau, t)$, and we assume that $\mathbb{T}$ is a periodic time scale under its matched space $(\mathbb{T}, \Pi, F, \delta)$. We will show that $\delta_{\tau}: \mathbb{T}^{*} \rightarrow \mathbb{T}^{*}$ are commutative with the forward jump operator $\sigma: \mathbb{T} \rightarrow \mathbb{T}$ given by $\sigma(t):=\inf \{s \in \mathbb{T}: s>t\}$, that is,

$$
\left(\delta_{\tau} \circ \sigma\right)(t)=\left(\sigma \circ \delta_{\tau}\right)(t)
$$

Lemma 2.21 The operator $\delta_{\tau}: \mathbb{T}^{*} \rightarrow \mathbb{T}^{*}$ preserves the structure of the points in $\mathbb{T}^{*}$. That is,

$$
\begin{gathered}
\sigma\left(t^{*}\right)=t^{*} \Rightarrow \sigma\left(\delta_{\tau}\left(t^{*}\right)\right)=\delta_{\tau}\left(t^{*}\right), \\
\sigma\left(t^{*}\right)>t^{*} \Rightarrow \sigma\left(\delta_{\tau}\left(t^{*}\right)\right)>\delta_{\tau}\left(t^{*}\right) .
\end{gathered}
$$

Proof Since $\sigma(t) \geq t$ for all $t \in \mathbb{T}^{*}$, thus, by $\left(P_{1}\right)$ from Definition 2.7, we obtain $\delta_{\tau}(\sigma(t)) \geq$ $\delta_{\tau}(t)$. Since $\sigma\left(\delta_{\tau}(t)\right)$ is the smallest element satisfying $\sigma\left(\delta_{\tau}(t)\right) \geq \delta_{\tau}(t)$, we obtain

$$
\delta_{\tau}(\sigma(t)) \geq \sigma\left(\delta_{\tau}(t)\right) \quad \text { for all } t \in \mathbb{T}^{*} .
$$


If $\sigma\left(t^{*}\right)=t^{*}$, then (5) implies $\delta_{\tau}\left(t^{*}\right)=\delta_{\tau}\left(\sigma\left(t^{*}\right)\right)$. That is, $\delta_{\tau}\left(t^{*}\right)=\sigma\left(\delta_{\tau}\left(t^{*}\right)\right)$.

If $\sigma\left(t^{*}\right)>t^{*}$, then by the definition of $\sigma$ we have

$$
\left(t^{*}, \sigma\left(t^{*}\right)\right) \cap \mathbb{T}^{*}=\emptyset,
$$

and by $\left(P_{1}\right)$ from Definition 2.7, $\delta_{\tau}\left(\sigma\left(t^{*}\right)\right)>\delta_{\tau}\left(t^{*}\right)$. For contradiction, assume that $\delta_{\tau}\left(t^{*}\right)$ is right-dense, i.e., $\sigma\left(\delta_{\tau}\left(t^{*}\right)\right)=\delta_{\tau}\left(t^{*}\right)$, and it follows from (5) that we have $\left(\delta_{\tau}\left(t^{*}\right), \delta_{\tau}\left(\sigma\left(t^{*}\right)\right)\right) \cap$ $\mathbb{T}^{*} \neq \emptyset$. Pick one element $s \in\left(\delta_{\tau}\left(t^{*}\right), \delta_{\tau}\left(\sigma\left(t^{*}\right)\right)\right) \cap \mathbb{T}^{*}$, and since $\delta_{\tau}(t)$ is strictly increasing in $t$, we obtain $\delta_{\tau}^{-1}(s) \in\left(t^{*}, \sigma\left(t^{*}\right)\right) \cap \mathbb{T}^{*}$, which contradicts (6). Therefore, $\delta_{\tau}\left(t^{*}\right)$ must be right-scattered, i.e., $\sigma\left(\delta_{\tau}\left(t^{*}\right)\right)>\delta_{\tau}\left(t^{*}\right)$. This completes the proof.

Corollary 2.22 For all $t \in \mathbb{T}^{*}$, the following are fulfilled:

$$
\begin{aligned}
& \delta_{\tau}(\sigma(t))=\sigma\left(\delta_{\tau}(t)\right) \\
& \delta_{\tau}^{-1}(\sigma(t))=\sigma\left(\delta_{\tau}^{-1}(t)\right) .
\end{aligned}
$$

Proof From the proof process of Lemma 2.21, we immediately obtain (7). By (7), we obtain that $\delta_{\tau}(\sigma(s))=\sigma\left(\delta_{\tau}(s)\right)$ for all $s \in \mathbb{T}^{*}$. Substituting $s=\delta_{\tau}^{-1}(t)$, we obtain $\delta_{\tau}\left(\sigma\left(\delta_{\tau}^{-1}(t)\right)\right)=$ $\sigma\left(\delta_{\tau}\left(\delta_{\tau}^{-1}(t)\right)\right)$, and it follows from (2) in Lemma 2.13 that $\delta_{\tau}^{-1}(\sigma(t))=\sigma\left(\delta_{\tau}^{-1}(t)\right)$ for all $t \in \mathbb{T}^{*}$. This completes the proof.

Observe that (7) along with (8) yields (4).

Definition 2.14 Let $\mathbb{T}$ be a periodic time scale under the matched space $(\mathbb{T}, \Pi, F, \delta)$ and $\mathbb{X}$ be a Banach space, and we say $f: \mathbb{T}^{*} \rightarrow \mathbb{X}$ is periodic under $(\mathbb{T}, \Pi, F, \delta)$ if there exists $\tau \in \Pi^{*}$ such that

$$
\left(\tau^{ \pm 1}, t\right) \in \mathcal{D}_{\delta} \quad \text { and } \quad f\left(\delta_{\tau^{ \pm 1}}(t)\right)=f(t) \quad \text { for all } t \in \mathbb{T}^{*}
$$

The number $\tau$ such that (9) holds is called the period of $f$.

Example 2.23 Let $\mathbb{T}=\mathbb{R}$ and $\Pi=[0,+\infty)$, and we define the following operators:

$$
\delta_{\tau}(t)=\left\{\begin{array}{ll}
\tau t, & \text { if } t \geq 0, \\
t / \tau, & \text { if } t<0,
\end{array} \quad \text { for } \tau \in[1,+\infty) \cap \Pi^{*}\right.
$$

and

$$
\delta_{\tau}^{-1}(t)=\left\{\begin{array}{ll}
t / \tau, & \text { if } t \geq 0, \\
\tau t, & \text { if } t<0,
\end{array} \quad \text { for } \tau \in[1,+\infty) \cap \Pi^{*},\right.
$$

and one easily observes that $(\mathbb{T}, \Pi, F, \delta)$ is a matched space for $\mathbb{T}$, where

$$
F(A)= \begin{cases}\frac{1}{1-A}, & A \leq 0 \\ 1+A, & A>0\end{cases}
$$


for all $A \in \mathbb{T}^{*}=\mathbb{R} \backslash\{0\}, \Pi^{*}=(0,+\infty)$. By Definition 2.14, we know that the set of reals $\mathbb{R}$ is periodic under the matched space $(\mathbb{T}, \Pi, F, \delta)$. The function

$$
f_{\tau}(t)=\cos \left(\frac{\ln |t|}{\ln (1 / \sqrt{\tau})} \pi\right), \quad \tau>\text { land } t \in \mathbb{T}^{*}=\mathbb{R} \backslash\{0\}
$$

is periodic under $(\mathbb{T}, \Pi, F, \delta)$ with the period $\tau=P^{2}, P>1$ since

$$
\begin{aligned}
& f_{\tau}\left(\delta_{\tau^{ \pm 1}}(t)\right)= \begin{cases}f_{\tau}\left(t P^{ \pm 2}\right), & \text { if } t \geq 0 \\
f_{\tau}\left(t / P^{ \pm 2}\right), & \text { if } t<0,\end{cases} \\
& =\cos \left(\frac{\ln |t| \pm 2 \ln (1 / P)}{\ln (1 / P)} \pi\right) \\
& =\cos \left(\frac{\ln |t|}{\ln (1 / P)} \pi \pm 2 \pi\right)=\cos \left(\frac{\ln |t|}{\ln (1 / P)} \pi\right)=f_{\tau}(t) .
\end{aligned}
$$

Example 2.24 The time scale $\overline{q^{\mathbb{Z}}}=\left\{q^{n}, n \in \mathbb{Z}\right.$ and $\left.q>1\right\} \cup\{0\}$ is periodic under the matched space $(\mathbb{T}, \Pi, F, \delta)$ with period $\tau=q^{4}$. The function defined by

$$
f(t)=[\theta(t)]^{\frac{\ln t}{\ln q}}, \quad \theta(t)=\left\{\begin{array}{ll}
1, & t>1, \\
-1, & 0 \leq t<1,
\end{array} \quad t \in q^{\mathbb{Z}}\right.
$$

is periodic with period $\tau=q^{4}$ since $\delta_{\tau^{ \pm 1}}(t)=q^{ \pm 4} t \in \overline{q^{\mathbb{Z}}} \backslash\{0\}=q^{\mathbb{Z}}$ and

$$
f\left(\delta_{\tau^{ \pm 1}}(t)\right)=[\theta(t)]^{\frac{\ln t}{\ln q} \pm 4}=[\theta(t)]^{\frac{\ln t}{\ln q}}=f(t)
$$

for all $t \in q^{\mathbb{Z}}$. However, $f$ is not periodic in the sense of Definition 1.1 since there is no positive number $\tau$ such that $f(t \pm \tau)=f(t)$ holds.

In the following, we introduce the concept of $\Delta$-periodic function under the matched space $(\mathbb{T}, \Pi, F, \delta)$.

Definition 2.15 Let $\mathbb{T}$ be a periodic time scale under the matched space $(\mathbb{T}, \Pi, F, \delta)$ and $\mathbb{X}$ be a Banach space, and we say that a function $f: \mathbb{T}^{*} \rightarrow \mathbb{X}$ is $\Delta$-periodic if there exists $\tau \in \Pi^{*}$ such that

$$
\left(\tau^{ \pm 1}, t\right) \in \mathcal{D}_{\delta} \quad \text { for all } t \in \mathbb{T}^{*}
$$

and the shifts $\delta_{\tau^{ \pm 1}}$ are $\Delta$-differentiable with rd-continuous derivatives and

$$
f\left(\delta_{\tau^{ \pm 1}}(t)\right) \delta_{\tau^{ \pm 1}}^{\Delta}(t)=f(t)
$$

for all $t \in \mathbb{T}^{*}$. The number $\tau$ is called the period of $f$.

Example 2.25 For any $a \in \mathbb{R} \backslash\{0\}$, the real-valued function $f(t)=a / t$ defined on $5^{\mathbb{Z}}=$ $\left\{5^{n}, n \in \mathbb{Z}\right\}$ is $\Delta$-periodic under the matched space $(\mathbb{T}, \Pi, F, \delta)$ with the period $\tau=5$ since

$$
f\left(\delta_{5^{ \pm 1}}(t)\right) \delta_{5^{ \pm 1}}^{\Delta}(t)=\frac{a}{5^{ \pm 1} t} 5^{ \pm 1}=\frac{a}{t}=f(t) .
$$


Theorem 2.26 Let $\mathbb{T}$ be a periodic function under the matched space $(\mathbb{T}, \Pi, F, \delta)$ and $f$ be a $\Delta$-periodic function with period $\tau \in \Pi^{*}$. Assume that $f \in C_{r d}(\mathbb{T})$. Then

$$
\int_{t_{0}}^{t} f(s) \Delta s=\int_{\delta_{\tau} \pm 1}^{\delta_{\tau} \pm 1}(t) f(s) \Delta s .
$$

Proof From Theorem 1.98 in [8], substituting $v(s)=\delta_{\tau}(s)$ and $g(s)=f\left(\delta_{\tau}(s)\right.$ ), we obtain that

$$
\begin{aligned}
\int_{\delta_{\tau}\left(t_{0}\right)}^{\delta_{\tau}(t)} f(s) \Delta s & =\int_{v\left(t_{0}\right)}^{v(t)} g\left(v^{-1}(s)\right) \Delta s \\
& =\int_{t_{0}}^{t} g(s) v^{\Delta}(s) \Delta s \\
& =\int_{t_{0}}^{t} f\left(\delta_{\tau}(s)\right) \delta_{\tau}^{\Delta}(s) \Delta s=\int_{t_{0}}^{t} f(s) \Delta s .
\end{aligned}
$$

The equality

$$
\int_{\delta_{\tau}^{-1}\left(t_{0}\right)}^{\delta_{\tau}^{-1}(t)} f(s) \Delta s=\int_{t_{0}}^{t} f(s) \Delta s, \quad \text { i.e., } \int_{\delta_{\tau^{-1}}\left(t_{0}\right)}^{\delta_{\tau^{-1}}(t)} f(s) \Delta s=\int_{t_{0}}^{t} f(s) \Delta s
$$

can be obtained similarly. The proof is complete.

Remark 2.27 From Definition 2.26, if the time scale is in the sense of Definition 1.1, then one can obtain $\int_{t_{0} \pm \tau}^{t \pm \tau} f(s) \Delta s=\int_{t_{0}}^{t} f(s) \Delta s$ by taking $\delta_{\tau^{ \pm 1}}(t)=t \pm \tau$.

In the following, according to Definitions 2.8 and 2.9, one can also introduce the concept of matched spaces for time scales under shift operators attached with the shift directions.

Definition 2.16 Let the pair $\left(\Pi^{*}, \tilde{\delta}\right)$ be closed towards the operation $\tilde{\delta}$, and $\Pi^{*}, \mathbb{T}^{*}$ be the largest subsets of the time scales $\Pi$ and $\mathbb{T}$, respectively. Further, let $\Pi$ be an adjoint set of $\mathbb{T}$ and $F$ be the adjoint mapping between $\mathbb{T}$ and $\Pi$. If there exists the shift operator $\delta$ satisfying Definition 2.8 , then we say the group $(\mathbb{T}, \Pi, F, \delta)$ is a matched space attached with the shift direction for $\mathbb{T}$, i.e., one can describe it as follows:

(i) if $\delta$ is a bi-direction shift operator, then we say $(\mathbb{T}, \Pi, F, \delta)$ is a bi-direction matched space;

(ii) if $\delta$ is a positive-direction shift operator, then we say $(\mathbb{T}, \Pi, F, \delta)$ is a positive-direction matched space;

(iii) if $\delta$ is a negative-direction shift operator, then we say $(\mathbb{T}, \Pi, F, \delta)$ is a negative-direction matched space;

(iv) a positive-direction or negative-direction matched space is called an oriented-direction matched space.

Remark 2.28 Note that the matched space $(\mathbb{T}, \Pi, F, \delta)$ established in the sense of Definition 2.7 is the bi-direction matched space since $\delta$ is the bi-direction shift operator.

Definition 2.17 Under Definition 2.16, a time scale $\mathbb{T}$ is called the complete closedness time scale under a matched space $(\mathbb{T}, \Pi, F, \delta)$ attached with the shift direction if

$$
\tilde{\Pi}:=\left\{\tau \in \Pi^{*}:(\tau, t) \in \mathcal{D}_{\delta}, \forall t \in \mathbb{T}^{*}\right\} \notin\left\{\left\{e_{\Pi^{*}}\right\}, \varnothing\right\} .
$$




\section{Moreover,}

(i) if $\tilde{\Pi}^{+}:=\tilde{\Pi} \cap\left[e_{\Pi^{*}},+\infty\right) \notin\left\{\left\{e_{\Pi^{*}}\right\}, \varnothing\right\}$, then we say $\mathbb{T}$ is a positive-direction complete closedness time scale;

(ii) if $\tilde{\Pi}^{-}:=\tilde{\Pi} \cap\left(-\infty, e_{\Pi^{*}}\right] \notin\left\{\left\{e_{\Pi^{*}}\right\}, \emptyset\right\}$, then we say $\mathbb{T}$ is a negative-direction complete closedness time scale;

(iii) if $\tilde{\Pi}^{ \pm}:=\tilde{\Pi}^{+} \cup \tilde{\Pi}^{-}$and $\tilde{\Pi}^{+}, \tilde{\Pi}^{-} \notin\left\{\left\{e_{\Pi^{*}}\right\}, \emptyset\right\}$, then we say $\mathbb{T}$ is a bi-direction complete closedness time scale;

(iv) a positive-direction or negative-direction complete closedness time scale is called an oriented-direction complete closedness time scale.

Remark 2.29 For Example 2.5, according to Definition 2.17, one can observe that $\mathbb{T}_{1}$ is a positive-direction complete closedness time scale under the matched space $\left(\mathbb{T}_{1}, \Pi_{1}, F_{1}, \delta_{1}\right)$, and $\mathbb{T}_{2}$ is a negative-direction complete closedness time scale under the matched space $\left(\mathbb{T}_{2}, \Pi_{2}, F_{2}, \delta_{2}\right)$. Moreover, a bi-direction complete closedness time scale is actually equivalent to the periodic time scale under Definition 2.13.

Remark 2.30 In Definition 2.17, one will observe that $\tilde{\Pi}^{+}$is a positive-direction semigroup and $\tilde{\Pi}^{-}$is a negative-direction semigroup according to Definition 2.10.

\section{Applications}

In this section, we introduce some new concepts of almost periodic functions and almost automorphic functions based on the algebraic structure of matched spaces for time scales. Throughout this section, we assume that $\mathbb{X}$ is a Banach space and $D \subseteq \mathbb{X}$ is an open set.

In the following, we assume that $(\mathbb{T}, \Pi, F, \delta)$ is a bi-direction matched space, then all the elements from $\Pi^{*}$ have the corresponding inverse elements in $\Pi^{*}$.

Definition 3.1 Let $\mathbb{T}$ be a periodic time scale under the matched space $(\mathbb{T}, \Pi, F, \delta)$. A function $f \in C(\mathbb{T} \times D, \mathbb{X})$ is called an almost periodic function with shift operators in $t \in \mathbb{T}$ uniformly for $x \in D$ if the $\varepsilon$-shift set of $f$

$$
E\{\varepsilon, f, S\}=\left\{\tau \in \tilde{\Pi}:\left\|f\left(\delta_{\tau^{ \pm 1}}(t), x\right)-f(t, x)\right\|<\varepsilon \text { for all } t \in \mathbb{T}^{*} \text { and } x \in S\right\}
$$

is a relatively dense set with respect to the pair $\left(\Pi^{*}, \tilde{\delta}\right)$ for all $\varepsilon>0$ and for each compact subset $S$ of $D$; that is, for any given $\varepsilon>0$ and each compact subset $S$ of $D$, there exists a constant $l(\varepsilon, S)>0$ such that each interval of length $l(\varepsilon, S)$ contains $\tau(\varepsilon, S) \in E\{\varepsilon, f, S\}$ such that

$$
\left\|f\left(\delta_{\tau^{ \pm 1}}(t), x\right)-f(t, x)\right\|<\varepsilon \quad \text { for all } t \in \mathbb{T}^{*} \text { and } x \in S .
$$

Now $\tau$ is called the $\varepsilon$-shift number of $f$ and $l(\varepsilon, S)$ is called the inclusion length of $E\{\varepsilon, f, S\}$.

Example 3.1 Based on the time scale in Example 2.23, consider the function

$$
\tilde{F}(t)=\cos \left(\frac{\ln |\sqrt{2} t|}{\ln \left(1 / P_{1}\right)} \pi\right)+\cos \left(\frac{\ln |\sqrt{3} t|}{\ln \left(1 / P_{2}\right)} \pi\right)
$$




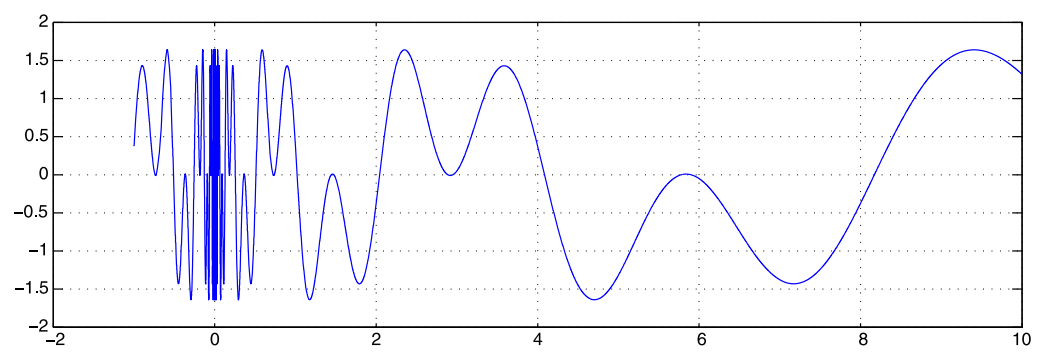

Figure 1 Graph of $\tilde{F}(t)=\cos \left(\frac{\ln |\sqrt{2} t|}{\ln \left(1 / P_{1}\right)} \pi\right)+\cos \left(\frac{\ln |\sqrt{3} t|}{\ln \left(1 / P_{2}\right)} \pi\right)$ with $P_{1}=2, P_{2}=\sqrt[3]{2}$.

where $P_{1} \neq P_{2}, P_{1}, P_{2}>1$ and $t \in \mathbb{T}^{*}=\mathbb{R} \backslash\{0\}$. One can observe that $\tilde{F}(t)$ is almost periodic under the matched space $(\mathbb{T}, \Pi, F, \delta)$. From Example 2.23, let

$$
f_{P_{1}^{2}}(\sqrt{2} t)=\cos \left(\frac{\ln |\sqrt{2} t|}{\ln \left(1 / P_{1}\right)} \pi\right), \quad f_{P_{2}^{2}}(\sqrt{3} t)=\cos \left(\frac{\ln |\sqrt{3} t|}{\ln \left(1 / P_{2}\right)} \pi\right)
$$

we obtain that $\tilde{F}(t)=f_{P_{1}^{2}}(\sqrt{2} t)+f_{P_{2}^{2}}(\sqrt{3} t)$, and note that $f_{P_{1}^{2}}$ and $f_{P_{2}^{2}}$ are periodic with different periods $P_{1}^{2}, P_{2}^{2}$, respectively (see Figure 1 ).

Remark 3.2 From Definition 3.1, one can easily establish the following classical concepts of almost periodic functions on $\mathbb{R}$ and $h \mathbb{Z}, h>0$, etc. Here we present some of them.

(i) Let $\mathbb{T}=\bigcup_{k \in \mathbb{Z}}[k(a+b), k(a+b)+b]$, where $a \neq-b$. Obviously, if $b=0, a=1$, then $\mathbb{T}=\mathbb{Z}$. If $b=1, a=0$, then $\mathbb{T}=\mathbb{R}$. Let $\Pi^{*}=\{n(a+b): n \in \mathbb{Z}\}$, then $\tilde{\Pi}=\Pi^{*}$ and $\tilde{\delta}\left(\tau_{1}, \tau_{2}\right)=\tau_{1}+\tau_{2}$, where $\tau_{1}, \tau_{2} \in \Pi^{*}$. Thus, let $\delta_{\tau^{ \pm 1}}(t)=t \pm \tau$, then we can easily obtain the following concept of almost periodic functions on this periodic time scale.

Definition 3.2 A function $f \in C(\mathbb{T} \times D, \mathbb{X})$ is called an almost periodic function in $t \in \mathbb{T}$ uniformly for $x \in D$ if for all $\varepsilon>0$ and for each compact subset $S$ of $D$, the $\varepsilon$-shift set of $f$

$$
E\{\varepsilon, f, S\}=\left\{\tau \in \tilde{\Pi}:\|f(t \pm \tau, x)-f(t, x)\|<\varepsilon \text { for all } t \in \mathbb{T}^{*} \text { and } x \in S\right\}
$$

is a relatively dense set with respect to the pair $\left(\Pi^{*},+\right)$.

(ii) Let $\mathbb{T}=\overline{q^{\mathbb{Z}}}$ and $\Pi^{*}=q^{\mathbb{Z}}$. Then it is easy to get $\tilde{\Pi}=\Pi^{*}=q^{\mathbb{Z}}, \tilde{\delta}\left(\tau_{1}, \tau_{2}\right)=\tau_{1} \cdot \tau_{2}$, where $\tau_{1}, \tau_{2} \in \Pi^{*}$. Now, let $\delta_{\tau^{ \pm 1}}(t)=t \tau^{ \pm 1}$, then we can easily obtain the following concept of almost periodic functions on the quantum time scale.

Definition 3.3 A function $f \in C\left(\overline{q^{\mathbb{Z}}} \times D, \mathbb{X}\right)$ is called an almost periodic function in $t \in \overline{q^{\mathbb{Z}}}$ uniformly for $x \in D$ if for all $\varepsilon>0$ and for each compact subset $S$ of $D$, the $\varepsilon$-shift set of $f$

$$
E\{\varepsilon, f, S\}=\left\{\tau \in \tilde{\Pi}:\left\|f\left(t \tau^{ \pm 1}, x\right)-f(t, x)\right\|<\varepsilon \text { for all } t \in q^{\mathbb{Z}} \text { and } x \in S\right\}
$$

is a relatively dense set with respect to the pair $\left(\Pi^{*}, \cdot\right)$. 
(iii) Let $\mathbb{T}=\mathbb{N}_{ \pm}^{\frac{1}{2}}$ and $\Pi^{*}=\mathbb{N}_{ \pm}^{\frac{1}{2}}$. Then we can easily obtain that $\tilde{\Pi}=\Pi^{*}=\mathbb{N}_{ \pm}^{\frac{1}{2}}$. Now let

$$
\begin{aligned}
& \delta_{\tau^{ \pm 1}}(t)= \begin{cases}\sqrt{t^{2}+\operatorname{sgn}( \pm \tau) \cdot \tau^{2}}, & t>0, \\
-\sqrt{t^{2}-\operatorname{sgn}( \pm \tau) \cdot \tau^{2}}, & t<0,\end{cases} \\
& \tilde{\delta}\left(\tau_{1}, \tau_{2}\right)= \begin{cases}\sqrt{\tau_{1}^{2}+\operatorname{sgn}\left(\tau_{2}\right) \cdot \tau_{2}^{2}}, & \tau_{1}>0, \\
-\sqrt{\tau_{1}^{2}-\operatorname{sgn}\left(\tau_{2}\right) \cdot \tau_{2}^{2}}, & \tau_{1}<0,\end{cases}
\end{aligned}
$$

where $\tau, \tau_{1}, \tau_{2} \in \Pi^{*}$. Hence, we can state the concept of almost periodic functions on $\mathbb{N}_{ \pm}^{\frac{1}{2}}$ as follows.

Definition 3.4 A function $f \in C\left(\mathbb{N}_{ \pm}^{\frac{1}{2}} \times D, \mathbb{X}\right)$ is called an almost periodic function in $t \in$ $\mathbb{N}_{ \pm}^{\frac{1}{2}}$ uniformly for $x \in D$ if for all $\varepsilon>0$ and for each compact subset $S$ of $D$, the $\varepsilon$-shift set of $f$

$$
E\{\varepsilon, f, S\}=\left\{\tau \in \tilde{\Pi}:\left\|f\left(\delta_{\tau^{ \pm 1}}(t), x\right)-f(t, x)\right\|<\varepsilon \text { for all } t \in \mathbb{N}_{ \pm}^{\frac{1}{2}} \text { and } x \in S\right\}
$$

is a relatively dense set with respect to the pair $\left(\Pi^{*}, \tilde{\delta}\right)$.

Note that Definition 3.2 is accurately suitable for any periodic time scale in the sense of Definition 1.1, which implies that the almost periodic functions investigated in [13, 14, 16-18] are just some particular cases of Definition 3.1. In fact, the almost periodic phenomenon reflected by Example 3.1 would be impossible to be studied in the previous literature works because there have been no concepts of almost periodic functions under non-translational shifts until now.

Remark 3.3 According to Definition 2.16, if we attach a shift direction to the matched space, then we can derive some concepts of almost periodic functions attached with shift directions. We present some of them here, the readers can also establish the similar concepts on other irregular time scales.

(i) Let $\mathbb{T}=\overline{\left\{q^{n}: q>1, n \in \mathbb{Z}^{+}\right\}}$and $\Pi^{*}=\left\{q^{n}: q>1, n \in \mathbb{Z}^{+}\right\} \cup\{1\}$. Then it is easy to get $\tilde{\Pi}=\Pi^{*}, \tilde{\delta}\left(\tau_{1}, \tau_{2}\right)=\tau_{1} \cdot \tau_{2}$, where $\tau_{1}, \tau_{2} \in \Pi^{*}$. Now, let $\delta_{\tau}(t)=t \tau$, then we can easily obtain the following concept of positive-direction almost periodic functions on the time scale $\mathbb{T}$.

Definition 3.5 A function $f \in C(\mathbb{T} \times D, \mathbb{X})$ is called a positive-direction almost periodic function in $t \in \mathbb{T}$ uniformly for $x \in D$ if for all $\varepsilon>0$ and for each compact subset $S$ of $D$, the $\varepsilon$-shift set of $f$

$$
E\{\varepsilon, f, S\}=\{\tau \in \tilde{\Pi}:\|f(t \tau, x)-f(t, x)\|<\varepsilon \text { for all } t \in \mathbb{T} \text { and } x \in S\}
$$

is a relatively dense set with respect to the pair $\left(\Pi^{*}, \cdot\right)$.

(ii) Let $\mathbb{T}=\overline{\left\{q^{n}: q>1, n \in \mathbb{Z}^{-}\right\}}$and $\Pi^{*}=\left\{q^{n}: q>1, n \in \mathbb{Z}^{-}\right\} \cup\{1\}$. Then it is easy to get $\tilde{\Pi}=\Pi^{*}, \tilde{\delta}\left(\tau_{1}, \tau_{2}\right)=\tau_{1} \cdot \tau_{2}$, where $\tau_{1}, \tau_{2} \in \Pi^{*}$. Now, let $\delta_{\tau}(t)=t \tau$, then we can easily obtain the following concept of negative-direction almost periodic functions on the time scale $\mathbb{T}$. 
Definition 3.6 A function $f \in C(\mathbb{T} \times D, \mathbb{X})$ is called a negative-direction almost periodic function in $t \in \mathbb{T}$ uniformly for $x \in D$ if for all $\varepsilon>0$ and for each compact subset $S$ of $D$, the $\varepsilon$-shift set of $f$

$$
E\{\varepsilon, f, S\}=\{\tau \in \tilde{\Pi}:\|f(t \tau, x)-f(t, x)\|<\varepsilon \text { for all } t \in \mathbb{T} \text { and } x \in S\}
$$

is a relatively dense set with respect to the pair $\left(\Pi^{*}, \cdot\right)$.

From Definitions 3.5 and 3.6, one can observe that $\left(\Pi^{*}, \tilde{\delta}\right)$ are not Abelian groups but are just a semigroup towards the operators $\tilde{\delta}$. We can unify them as follows.

Definition 3.7 Let $\mathbb{T}$ be an oriented-direction complete closedness time scale under the oriented-direction matched space $(\mathbb{T}, \Pi, F, \delta)$. A function $f \in C(\mathbb{T} \times D, \mathbb{X})$ is called an almost periodic function with shift operators in $t \in \mathbb{T}$ uniformly for $x \in D$ if for all $\varepsilon>0$ and for each compact subset $S$ of $D$, the $\varepsilon$-shift set of $f$

$$
E\{\varepsilon, f, S\}=\left\{\tau \in \tilde{\Pi}:\left\|f\left(\delta_{\tau}(t), x\right)-f(t, x)\right\|<\varepsilon \text { for all } t \in \mathbb{T}^{*} \text { and } x \in S\right\}
$$

is a relatively dense set with respect to the pair $\left(\Pi^{*}, \tilde{\delta}\right)$. Moreover,

(i) if $(\mathbb{T}, \Pi, F, \delta)$ is a positive-direction matched space (or $\Pi^{*}$ is a positive-direction semigroup), then $f$ is called the positive-direction almost periodic function;

(ii) if ( $\mathbb{T}, \Pi, F, \delta)$ is a negative-direction matched space (or $\Pi^{*}$ is a negative-direction semigroup), then $f$ is called the negative-direction almost periodic function;

(iii) if ( $\mathbb{T}, \Pi, F, \delta)$ is a bi-direction matched space (or $\Pi^{*}$ is an Abelian group), then $f$ is called the bi-direction almost periodic function.

In the sequel, for simplicity and comprehensive statement, we assume that a matched space is an oriented-direction matched space, we can extend Definition 3.7 to the orienteddirection $\Delta$-almost periodic functions.

Definition 3.8 Let $\mathbb{T}$ be an oriented-direction complete closedness time scale under the matched space $(\mathbb{T}, \Pi, F, \delta)$, the shift $\delta_{\tau}(t)$ is $\Delta$-differentiable with $r d$-continuous bounded derivatives $\delta_{\tau}^{\Delta}(t):=\delta^{\Delta}(\tau, t)$ for all $t \in \mathbb{T}^{*}$. A function $f \in C(\mathbb{T} \times D, \mathbb{X})$ is called an almost periodic function with shift operators in $t \in \mathbb{T}$ uniformly for $x \in D$ if the $\varepsilon$-shift set of $f$

$$
E\{\varepsilon, f, S\}=\left\{\tau \in \tilde{\Pi}:\left\|f\left(\delta_{\tau}(t), x\right) \delta_{\tau}^{\Delta}(t)-f(t, x)\right\|<\varepsilon \text { for all } t \in \mathbb{T}^{*} \text { and } x \in S\right\}
$$

is a relatively dense set with respect to the pair $\left(\Pi^{*}, \tilde{\delta}\right)$ for all $\varepsilon>0$ and for each compact subset $S$ of $D$; that is, for any given $\varepsilon>0$ and each compact subset $S$ of $D$, there exists a constant $l(\varepsilon, S)>0$ such that each interval of length $l(\varepsilon, S)$ contains $\tau(\varepsilon, S) \in E\{\varepsilon, f, S\}$ such that

$$
\left\|f\left(\delta_{\tau}(t), x\right) \delta_{\tau}^{\Delta}(t)-f(t, x)\right\|<\varepsilon \quad \text { for all } t \in \mathbb{T}^{*} \text { and } x \in S .
$$

Now $\tau$ is called the $\varepsilon$-shift number of $f$ and $l(\varepsilon, S)$ is called the inclusion length of $E\{\varepsilon, f, S\}$. Moreover, 
(i) if ( $\mathbb{T}, \Pi, F, \delta)$ is a positive-direction matched space (or $\Pi^{*}$ is a positive-direction semigroup), then $f$ is called the positive-direction $\Delta$-almost periodic function;

(ii) if $(\mathbb{T}, \Pi, F, \delta)$ is a negative-direction matched space (or $\Pi^{*}$ is a negative-direction semigroup), then $f$ is called the negative-direction $\Delta$-almost periodic function;

(iii) if ( $\mathbb{T}, \Pi, F, \delta)$ is a bi-direction matched space (or $\Pi^{*}$ is an Abelian group), then $f$ is called the bi-direction $\Delta$-almost periodic function.

Example 3.4 On the time scale $5^{\mathbb{Z}}=\left\{5^{n}, n \in \mathbb{Z}\right\}$, let $a, b \in \mathbb{R} \backslash\{0\}, a \neq b$ and

$$
g_{1}(t)=\frac{a}{t}, \quad g_{2}(t)=\frac{b}{(-1)^{\log _{5} t} t}, \quad \tilde{G}(t)=g_{1}(t)+g_{2}(t)=\frac{a}{t}+\frac{b}{(-1)^{\log _{5} t} t}
$$

from Example 2.25, one observes that $g_{1}\left(\delta_{5^{ \pm 1}}(t)\right) \delta_{5^{ \pm 1}}^{\Delta}(t)=g_{1}(t)$ and we note that

$$
\begin{aligned}
g_{2}\left(\delta_{5^{ \pm 2}}(t)\right)\left(\delta_{5^{ \pm 2}}\right)^{\Delta}(t) & =\frac{b}{(-1)^{\log _{5} 5^{ \pm 2} t} \cdot 5^{ \pm 2} t} \cdot 5^{ \pm 2} \\
& =\frac{b}{(-1)^{ \pm 2+\log _{5} t} \cdot t} \\
& =\frac{b}{(-1)^{\log _{5} t} t}=g_{2}(t) .
\end{aligned}
$$

Hence, $\tilde{G}(t)$ is a $\Delta$-almost periodic function under the matched space $(\mathbb{T}, \Pi, F, \delta)$.

Remark 3.5 The $\Delta$-almost periodicity from Definition 3.8 mainly focuses on the almost periodic functions on the "quantum-like" time scales such as $q^{\mathbb{Z}},(-q)^{\mathbb{Z}}$ and $\overline{-q^{\mathbb{Z}} \cup q^{\mathbb{Z}}}$, etc. Note that the almost periodicity and the $\Delta$-almost periodicity are equivalent on periodic time scales defined by Definition 1.1 since $\delta_{\tau}^{\Delta}(t)=(t+\tau)^{\Delta} \equiv 1$. Hence, the almost periodicity investigated in $[13,14,16-18]$ is actually equivalent to the $\Delta$-almost periodicity.

Next, we will introduce the concept of almost automorphic functions under bi-direction matched spaces for time scales.

Definition 3.9 Let $\mathbb{T}$ be a periodic time scale under the matched space $(\mathbb{T}, \Pi, F, \delta)$.

(i) Let $f: \mathbb{T} \rightarrow \mathbb{X}$ be a bounded continuous function. We say that $f$ is almost automorphic if from every sequence $\left\{s_{n}\right\} \subset \tilde{\Pi}$, we can extract a subsequence $\left\{\tau_{n}\right\}_{n=1}^{\infty}$ such that

$$
g(t)=\lim _{n \rightarrow \infty} f\left(\delta_{\tau_{n}}(t)\right)
$$

is well defined for each $t \in \mathbb{T}^{*}$ and

$$
\lim _{n \rightarrow \infty} g\left(\delta_{\tau_{n}^{-1}}(t)\right)=\lim _{n \rightarrow \infty} g\left(\delta_{\tau_{n}}^{-1}(t)\right)=f(t)
$$

for each $t \in \mathbb{T}^{*}$. Denote by $A A_{\delta}(\mathbb{T}, \mathbb{X})$ the set of all such functions.

(ii) A continuous function $f: \mathbb{T} \times \mathbb{X} \rightarrow \mathbb{X}$ is said to be almost automorphic if $f(t, x)$ is almost automorphic in $t \in \mathbb{T}^{*}$ uniformly for $x \in B$, where $B$ is any bounded subset of $\mathbb{X}$. Denote by $A A_{\delta}(\mathbb{T} \times \mathbb{X}, \mathbb{X})$ the set of all such functions. 


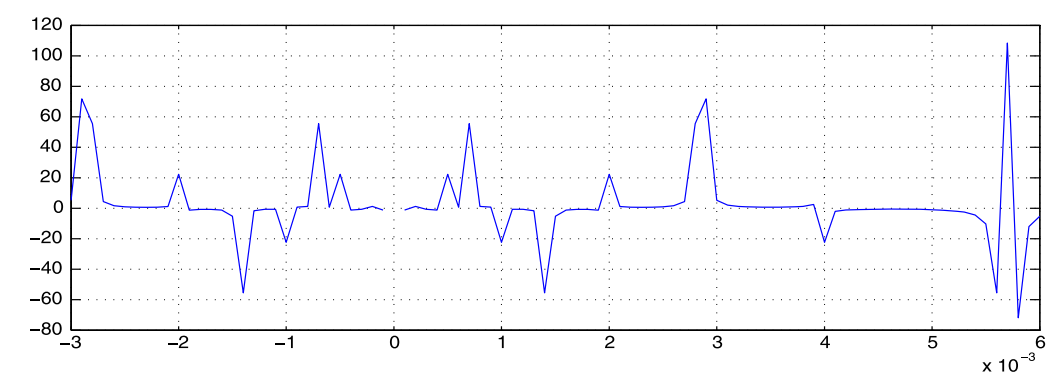

Figure 2 Graph of $\hat{F}(t)=\frac{1}{\cos \left(\frac{\ln |\sqrt{2} t|}{\ln \left(1 / P_{1}\right)} \pi\right)+\cos \left(\frac{\ln |\sqrt{3} t|}{\ln \left(1 / P_{2}\right)} \pi\right)}$ with $P_{1}=2, P_{2}=\sqrt[3]{2}$

Example 3.6 Recall Example 3.1 and consider the function

$$
\hat{F}(t)=1 /\left[\cos \left(\frac{\ln |\sqrt{2} t|}{\ln \left(1 / P_{1}\right)} \pi\right)+\cos \left(\frac{\ln |\sqrt{3} t|}{\ln \left(1 / P_{2}\right)} \pi\right)\right],
$$

where $P_{1} \neq P_{2}, P_{1}, P_{2}>1$ and $t \in \mathbb{T}^{*}=\mathbb{R} \backslash\{0\}$. One can observe that $\hat{F}(t)$ is almost automorphic under the matched space $(\mathbb{T}, \Pi, F, \delta)$. From Example 3.1, we can obtain that $\hat{F}(t)=\frac{1}{f_{P_{1}^{2}}(\sqrt{2} t)+f_{P_{2}^{2}}(\sqrt{3} t)}$ (see Figure 2).

Remark 3.7 Note that Definition 3.9 is the concept of bi-direction almost automorphic functions under a bi-direction matched space. Moreover, the almost automorphy reflected by Example 3.6 has never been considered in any literature until now. This is a completely new almost automorphic function under non-translational shifts.

Remark 3.8 If we attach the shift direction to a matched space, then one can obtain the following oriented-direction almost automorphic functions.

Definition 3.10 Let $\mathbb{T}$ be an oriented-direction complete closedness time scale under the oriented-direction matched space $(\mathbb{T}, \Pi, F, \delta)$.

(i) Let $f: \mathbb{T} \rightarrow \mathbb{X}$ be a bounded continuous function. We say that $f$ is oriented-direction almost automorphic if from every sequence $\left\{s_{n}\right\} \subset \tilde{\Pi}$, we can extract a subsequence $\left\{\tau_{n}\right\}_{n=1}^{\infty}$ such that

$$
g(t)=\lim _{n \rightarrow \infty} f\left(\delta_{\tau_{n}}(t)\right)
$$

is well defined for each $t \in \mathbb{T}^{*}$.

(ii) A continuous function $f: \mathbb{T} \times \mathbb{X} \rightarrow \mathbb{X}$ is said to be oriented-direction almost automorphic if $f(t, x)$ is oriented-direction almost automorphic in $t \in \mathbb{T}^{*}$ uniformly for $x \in B$, where $B$ is any bounded subset of $\mathbb{X}$.

We can extend Definition 3.9 to bi-direction $\Delta$-almost automorphic functions.

Definition 3.11 Let $\mathbb{T}$ be a periodic time scale under the matched space $(\mathbb{T}, \Pi, F, \delta)$.

(i) Let $f: \mathbb{T} \rightarrow \mathbb{X}$ be a bounded continuous function and the shift $\delta_{\tau}(t)$ is $\Delta$-differentiable with $r d$-continuous bounded derivatives $\delta_{\tau}^{\Delta}(t):=\delta^{\Delta}(\tau, t)$ for all 
$t \in \mathbb{T}^{*}$. We say that $f$ is $\Delta$-almost automorphic if from every sequence $\left\{s_{n}\right\} \subset \tilde{\Pi}$, we can extract a subsequence $\left\{\tau_{n}\right\}_{n=1}^{\infty}$ such that

$$
g(t)=\lim _{n \rightarrow \infty} f\left(\delta_{\tau_{n}}(t)\right) \delta_{\tau_{n}}^{\Delta}(t)
$$

is well defined for each $t \in \mathbb{T}^{*}$ and

$$
\lim _{n \rightarrow \infty} g\left(\delta_{\tau_{n}^{-1}}(t)\right) \delta_{\tau_{n}^{-1}}^{\Delta}(t)=f(t)
$$

for each $t \in \mathbb{T}^{*}$. Denote by $A A_{\delta}(\mathbb{T}, \mathbb{X})$ the set of all such functions.

(ii) A continuous function $f: \mathbb{T} \times \mathbb{X} \rightarrow \mathbb{X}$ is said to be $\Delta$-almost automorphic if $f(t, x)$ is $\Delta$-almost automorphic in $t \in \mathbb{T}^{*}$ uniformly for $x \in B$, where $B$ is any bounded subset of $\mathbb{X}$. Denote by $A A_{\delta}(\mathbb{T} \times \mathbb{X}, \mathbb{X})$ the set of all such functions.

Example 3.9 Recall Example 3.4 and, on the time scale $5^{\mathbb{Z}}=\left\{5^{n}, n \in \mathbb{Z}\right\}$, consider the following function:

$$
\hat{G}(t)=1 /\left[\frac{a}{t}+\frac{b}{(-1)^{\log _{5} t}}\right], \quad a, b \in \mathbb{R} \backslash\{0\}, a \neq b .
$$

One can observe that $\hat{G}(t)$ is almost automorphic under the matched space $(\mathbb{T}, \Pi, F, \delta)$. From Example 3.4, we can obtain that $\hat{G}(t)=\frac{1}{\tilde{G}(t)}$.

Remark 3.10 It is obvious that Definition 3.11 is the concept of bi-direction $\Delta$-almost automorphic functions under a bi-direction matched space. Note that $\Delta$-almost automorphy of functions mainly focuses on almost automorphic functions on the "quantum-like" time scales such as $q^{\mathbb{Z}},(-q)^{\mathbb{Z}}$ and $\overline{-q^{\mathbb{Z}} \cup q^{\mathbb{Z}}}$, etc. Moreover, the almost automorphy and $\Delta$ almost automorphy are equivalent on periodic time scales defined by Definition 1.1 since $\delta_{\tau}^{\Delta}(t)=(t+\tau)^{\Delta} \equiv 1$.

On the other hand, for $\nabla$-calculus theory is an analogy of $\Delta$-calculus theory on time scales, all the results established in this paper can be naturally extended to " $\nabla$-cases". Similar to Definition 3.11, one can also adopt the same idea to introduce the concept of $\nabla$ almost automorphic functions. Furthermore, the almost automorphy and the $\nabla$-almost automorphy are also equivalent on periodic time scales defined by Definition 1.1 since $\delta_{\tau}^{\nabla}(t)=(t+\tau)^{\nabla} \equiv 1$. Hence, the almost automorphy investigated in [12] is actually equivalent to the $\nabla$-almost automorphy.

Remark 3.11 Similar to Definition 3.10, one can also introduce the concept of $\Delta$-almost automorphic functions attached with shift directions as follows.

Definition 3.12 Let $\mathbb{T}$ be an oriented-direction complete closedness time scale under the oriented-direction matched space $(\mathbb{T}, \Pi, F, \delta)$.

(i) Let $f: \mathbb{T} \rightarrow \mathbb{X}$ be a bounded continuous function and the shift $\delta_{\tau}(t)$ is $\Delta$-differentiable with $r d$-continuous bounded derivatives $\delta_{\tau}^{\Delta}(t):=\delta^{\Delta}(\tau, t)$ for all $t \in \mathbb{T}^{*}$. We say that $f$ is oriented-direction $\Delta$-almost automorphic if from every 
sequence $\left\{s_{n}\right\} \subset \tilde{\Pi}$, we can extract a subsequence $\left\{\tau_{n}\right\}_{n=1}^{\infty}$ such that

$$
g(t)=\lim _{n \rightarrow \infty} f\left(\delta_{\tau_{n}}(t)\right) \delta_{\tau_{n}}^{\Delta}(t)
$$

is well defined for each $t \in \mathbb{T}^{*}$.

(ii) A continuous function $f: \mathbb{T} \times \mathbb{X} \rightarrow \mathbb{X}$ is said to be oriented-direction $\Delta$-almost automorphic if $f(t, x)$ is oriented-direction $\Delta$-almost automorphic in $t \in \mathbb{T}^{*}$ uniformly for $x \in B$, where $B$ is any bounded subset of $\mathbb{X}$.

Remark 3.12 Because an oriented-direction matched space is either positive-direction or negative-direction, then Definitions 3.10 and 3.12 can be attached with specific shift direction to describe the concept of almost automorphic functions and $\Delta$-almost automorphic functions with specific directions, respectively. It is easy to derive them by substituting a specific direction for "oriented-direction" in these two definitions, so we omit them here.

\section{Conclusion and discussion}

This paper is concerned with the problems of complete closedness of time scales under translational and non-translational shifts. By introducing the algebraic structure of matched spaces attached with shift directions, some basic concepts of complete closedness of time scales with different shift directions are introduced. Based on these, some new definitions of almost periodic functions and almost automorphic functions including their generalizations such as $\Delta$-almost periodic functions and $\Delta$-almost automorphic functions on irregular time scales, are proposed. Moreover, some novel almost periodicity and almost automorphy of some functions under non-translational shift are proposed and studied, which would be impossible to be investigated on periodic time scales under translations before. The results established in this paper will mainly contribute to solving the problems of closedness of time scales under non-translational shifts, which will be significant to studying functions on a more comprehensive scope of time scales such as the "quantum-like" time scales, e.g., $q^{\mathbb{Z}}, \overline{(-q)^{\mathbb{Z}}}, \overline{-q^{\mathbb{Z}} \cup q^{\mathbb{Z}}}$ and other irregular time scales, e.g., $\mathbb{N}_{+}^{\frac{1}{2}}, \mathbb{N}_{ \pm}^{\frac{1}{2}}$, etc.

As an important type of functions defined by translations, almost periodic functions on time scales have been paid much attention to. In the literature works [11,22], the authors studied almost periodic solutions of differential equations on certain time scales with transition conditions (DETC) on the basis of reduction to the impulsive differential equations (ITE). The authors employed the reversible translation transform of time scales to turn the time scale into the real line, then the differential equations on time scales can be changed into a traditional impulsive differential equations on $\mathbb{R}$ simultaneously. Finally, they established an equivalence between DETC and traditional ITE on $\mathbb{R}$. Their results mainly contribute to building a relationship between DETC and ITE, and through investigating the almost periodic solutions for ITE, the corresponding almost periodic solutions for DETC can be indirectly studied. However, they did not consider to directly use the $\Delta$-calculus theory on time scales to solve the almost periodic problems of differential equations on time scales since many time scales are not completely closed under translations, which will lead to the functions defined by translations being not well defined on time scales. Therefore, it was difficult to consider almost periodic problem on time scales directly through $\Delta$-calculus theory then. 
As a matter of fact, although $\Delta$-calculus theory on time scales has some similarity to Riemann calculus on $\mathbb{R}$, it has actually much more generality in calculus theory and more applications in the real world. The diversity of time variable form of time scales fully demonstrates huge essential differences between $\Delta$-calculus theory and Riemann calculus theory on $\mathbb{R}$ (see $[8,9])$. Moreover, $\Delta$-calculus theory will play a very important role in studying some classical problems which are in between the discrete time scale and the real line. Therefore, it is necessary and significant to employ $\Delta$-calculus theory to directly establish some related timescale theory on some classical problems. To some extent, it is insufficient and circumscribed to solve the problems only through building the relationship between some time scale and $\mathbb{R}$ since this relationship may be difficult to be established, or it does not exist (because some results established on $\mathbb{R}$ are not suitable for time scales), or it is particular (because if the relationship could be built, it will indicate that some results established on $\mathbb{R}$ are suitable for the time scale). For instance, if the graininess function $\mu$ of a time scale satisfies $\mu(t) \rightarrow+\infty$ as $t \rightarrow+\infty$, then the $\psi$-substitutions from [11] and [22] are ineffective, which implies that the results from [11] and [22] cannot be applied to the following types of time scales:

(1) $\mathbb{T}=\overline{q^{\mathbb{Z}}}$, it follows that $\mu(t) \rightarrow+\infty$ as $t \rightarrow+\infty$;

(2) $\mathbb{T}=\mathbb{N}_{0}^{2}=\left\{n^{2}, n \in \mathbb{N}_{0}\right\}$, it follows that $\mu(t) \rightarrow+\infty$ as $t \rightarrow+\infty$;

(3) $\mathbb{T}=-q^{\mathbb{Z}} \cup q^{\mathbb{Z}} \cup \mathbb{N}_{0}^{2} \cup \mathbb{N}_{0}^{3}$, it follows that $\mu(t) \rightarrow+\infty$ as $t \rightarrow+\infty$.

Note that in the literature [11], the $\psi$-substitution is

$$
\psi(t)=\left\{\begin{array}{ll}
t-\sum_{0<t_{2 k}<t} \delta_{k}, & t \geq 0, \\
t+\sum_{t \leq t_{2 k}<0} \delta_{k}, & t<0,
\end{array} \quad \text { where } \delta_{k}=t_{2 k+1}-t_{2 k} .\right.
$$

Obviously, such a $\psi$-substitution is a linear translation transform essentially, it is unsuitable for time scales (1), (2), (3) under non-translational shifts because all the points are isolated and the shifts of time scales (1), (2), (3) are not translational when considering almost periodic problems on them. In fact, there are many time scales which have a similar property as the above examples. Therefore, it is better to establish the corresponding timescale theory on some classical problems directly through $\Delta$-calculus theory on time scales. By using the results in this paper, the problem can be considered on more irregular time scales including some time scales with $\mu(t) \rightarrow+\infty$ as $t \rightarrow+\infty$.

On the other hand, the occurrence and change of many natural phenomena may directly depend on time scales, but not depend on the real line. The period of the occurrence and change of these phenomena can be directly and accurately described by the time scale rather than the real line. For this, Wang, Agarwal and O'Regan proposed the concept of time scales with almost periodicity and studied the double-almost periodic solutions for dynamic equations (see [23-25]) since the recurrence of many natural phenomena may take approximative periods of time rather than absolutely equivalent time intervals. Therefore, it is more practical to consider dynamic equations on almost periodic time scales by using $\Delta$-calculus theory. To investigate some important problems on time scales directly, the important and first step is to establish well-defined classical functions on time scales. In the literature [16, 17], the authors solved the problems of closedness of time scales under translations and introduced some concepts of almost periodic functions defined by translations, which realized that one is able to solve almost periodic problems through $\Delta$-calculus theory directly without considering any relationship 
between the time scale and $\mathbb{R}$. Moreover, in the literature [18], the authors introduced the concept of changing-periodic time scales and proposed the decomposition theorem of time scales to decompose an arbitrary time scale with the bounded graininess function $\mu$ into countable periodic time scales attached with directions, which will guarantee the local complete closedness of a time scale with the bounded graininess function $\mu$ under translations. Then the authors made the important supplements and comments on changing-periodic time scales and almost periodic functions (see [17, 21]). In this work, the authors construct the algebraic structure of matched spaces to solve the closedness of time scales under non-translational shifts, which lays a foundation for considering almost periodic problems, almost automorphic problems and other related topics for dynamic equations on irregular time scales by directly using $\Delta$-calculus theory in the future.

\section{Acknowledgements}

We express our sincere thanks to all the reviewers' insightful comments to improve this manuscript. This work was done while Chao Wang was a visiting scholar at Texas A\&M University-Kingsville. This work is supported by Youth Fund of NSFC (No. 11601470), Tian Yuan Fund of NSFC (No. 11526181), Yunnan Province Science and Technology Department Applied Basic Research Project of China (No. 2014FB102), and Dong Lu Youth Excellent Teachers Development Program of Yunnan University (No. wx069051).

\section{Competing interests}

The authors declare that they have no competing interests.

\section{Authors' contributions}

All authors contributed equally to the manuscript and typed, read and approved the final manuscript.

\section{Author details}

${ }^{1}$ Department of Mathematics, Yunnan University, Kunming, Yunnan 650091, People's Republic of China. ${ }^{2}$ Department of Mathematics, Texas A\&M University-Kingsville, 700 University Blvd., Kingsville, TX 78363-8202, USA. ${ }^{3}$ School of Mathematics, Statistics and Applied Mathematics, National University of Ireland, Galway, Ireland.

\section{Publisher's Note}

Springer Nature remains neutral with regard to jurisdictional claims in published maps and institutional affiliations.

Received: 1 June 2017 Accepted: 19 September 2017 Published online: 02 October 2017

\section{References}

1. Fink, M: Almost Periodic Differential Equations. Springer, Berlin (1974)

2. Diagana, T: Weighted pseudo almost periodic functions and applications. C. R. Math. 343, 643-646 (2006)

3. Diagana, T: Existence of pseudo-almost automorphic solutions to some abstract differential equations with $S^{p}$-pseudo-almost automorphic coefficients. Nonlinear Anal., Theory Methods Appl. 70, 3781-3790 (2009)

4. Zhang, C: Pseudo almost periodic solutions of some differential equations. J. Math. Anal. Appl. 181, $62-76$ (1994)

5. Zhang, C: Pseudo almost periodic solutions of some differential equations II. J. Math. Anal. Appl. 192, $543-561$ (1995)

6. Liang, J, Zhang, J, Xiao, T: Composition of pseudo almost automorphic and asymptotically almost automorphic functions. J. Math. Anal. Appl. 340, 1493-1499 (2008)

7. Hilger, S: Ein Maßkettenkalkül mit Anwendung auf Zentrumsmannigfaltigkeiten. PhD Thesis, Universität. Würzburg. 1988 (in German)

8. Bohner, M, Peterson, A: Dynamic Equations on Time Scales, an Introduction with Applications. Birkhäuser, Boston (2001)

9. Agarwal, RP, Bohner, M: Basic calculus on time scales and some of its applications. Results Math. 35, 3-22 (1999)

10. Kaufmann, ER, Raffoul, YN: Periodic solutions for a neutral nonlinear dynamical equation on a time scale. J. Math. Anal. Appl. 319, 315-325 (2006)

11. Akhmet, MU, Turan, M: The differential equations on time scales through impulsive differential equations. Nonlinear Anal. TMA 65, 2043-2060 (2006)

12. Wang, C, Agarwal, RP: Weighted piecewise pseudo almost automorphic functions with applications to abstract impulsive $\nabla$-dynamic equations on time scales. Adv. Differ. Equ. 2014, 153 (2014)

13. Wang, C, Agarwal, RP: Uniformly rd-piecewise almost periodic functions with applications to the analysis of impulsive $\Delta$-dynamic system on time scales. Appl. Math. Comput. 259, 271-292 (2015)

14. Wang, C: Almost periodic solutions of impulsive BAM neural networks with variable delays on time scales. Commun. Nonlinear Sci. Numer. Simul. 19, 2828-2842 (2014)

15. Adivar, M: A new periodic concept for time scales. Math. Slovaca 63, 817-828 (2013)

16. Wang, C, Agarwal, RP: Relatively dense sets, corrected uniformly almost periodic functions on time scales, and generalizations. Adv. Differ. Equ. 2015, 312 (2015)

17. Wang, C, Agarwal, RP, O'Regan, D: Periodicity, almost periodicity for time scales and related functions. Nonauton. Dyn. Syst. 3, 24-41 (2016) 
18. Wang, C, Agarwal, RP: Changing-periodic time scales and decomposition theorems of time scales with applications to functions with local almost periodicity and automorphy. Adv. Differ. Equ. 2015, 296 (2015)

19. Wang, C, Agarwal, RP: Almost periodic solution for a new type of neutral impulsive stochastic Lasota-Wazewska timescale model. Appl. Math. Lett. 70, 58-65 (2017)

20. Wang, C, Agarwal, RP, Sakthivel, R: Almost periodic oscillations for delay impulsive stochastic Nicholson's blowflies timescale model. Comput. Appl. Math. (2017). doi:10.1007/s40314-017-0495-0

21. Agarwal, RP, O'Regan, D: Some comments and notes on almost periodic functions and changing-periodic time scales. Electron. J. Math. Anal. Appl. 6, 125-136 (2018)

22. Akhmet, MU, Turan, M: Differential equations on variable time scales. Nonlinear Anal., Theory Methods Appl. 70 1175-1192 (2009)

23. Wang, C, Agarwal, RP, O'regan, D: Compactness criteria and new impulsive functional dynamic equations on time scales. Adv. Differ. Equ. 2016, 197 (2016)

24. Wang, C, Agarwal, RP, O'regan, D: Weighted piecewise pseudo double-almost periodic solution for impulsive evolution equations. J. Nonlinear Sci. Appl. 10, 3863-3886 (2017)

25. Wang, C, Agarwal, RP: Almost periodic dynamics for impulsive delay neural networks of a general type on almost periodic time scales. Commun. Nonlinear Sci. Numer. Simul. 36, 238-251 (2016)

\section{Submit your manuscript to a SpringerOpen ${ }^{\circ}$ journal and benefit from:}

- Convenient online submission

- Rigorous peer review

- Open access: articles freely available online

- High visibility within the field

- Retaining the copyright to your article

Submit your next manuscript at $\boldsymbol{\nabla}$ springeropen.com 